\title{
Design and Development of a Self-contained and Non-Invasive Integrated System for Electricity Monitoring Applications
}

by

\section{Sid Zarabi}

\author{
A thesis \\ presented to the University of Waterloo \\ in fulfillment of the \\ thesis requirement for the degree of \\ Master of Applied Science \\ In \\ Electrical and Computer Engineering
}

Waterloo, Ontario, Canada, 2017

(C) Sid Zarabi 2017 
I hereby declare that I am the sole author of this thesis. This is a true copy of the thesis, including any required final revisions, as accepted by my examiners.

I understand that my thesis may be made electronically available to the public. 


\begin{abstract}
Growing interest in improving the energy consumption efficiency in residential and commercial buildings has led to the emergence of intelligent energy management systems. This growing technology allows the transformation of the outdated electric distribution network within buildings to a smart and intelligent system. A major challenge in the development of such infrastructure is the need for low cost, integrated, self-contained, and non-invasive wireless sensor nodes. While an electric meter provides the utility company with information regarding the total energy consumption, no information is provided to the consumers regarding the energy consumed by individual appliances. Such visibility can provide consumers with the ability to better control and manage their energy usage leading to a reduced overall energy consumption.

This work explores the design and development of a self-contained and non-invasive integrated system intended for real-time electricity monitoring within residential and commercial buildings. The proposed system includes an Energy Harvester, an electric current sensor, a Micro-controller Unit, and a wireless communication device. The proposed system is self-powered and non-invasive, which offers a promising solution in providing real time information regarding the energy distribution within buildings.

The design featured in this work provides an innovative approach in the development of a customized interface circuitry that is designed to collect and regulate the energy from the Energy Harvester. The entire sensor node will operate under a power budget in the range of microwatts collected by the Energy Harvester. A Wireless MCU is programmed to acquire, process, and transmit the data from the sensor to the central hub via Bluetooth Low Energy connectivity. The real-time data transmitted to the central hub provides detailed information regarding the energy consumed by individual appliances within the building.
\end{abstract}




\section{Acknowledgements}

This dissertation would not have materialized without the encouragement, help, and guidance of countless people who have offered me their unfailing support. Without their help, it would have been impossible to accomplish this work.

First and foremost, I owe a great debt of gratitude to my supervisor, Dr. Lan Wei, who provided me with the opportunity to work on this project. For her unwavering encouragement and support whilst allowing me the room to work in my own way. Our meetings always brought new energy in me and left me with a glimpse of hope. To work with you has been a real pleasure to me and I will always remember your kind personality.

I owe the greatest thanks to my co-supervisor, Dr. David Nairn, for his endless encouragement and full support from the very early stages of this research project. For his remarkable ways in making the most complicated circuit concepts easy to understand. Many times I entered his office dejected and frustrated, not able to get the results I was looking for. His incredible use of analogies to compare abstract circuit problems to simple real life examples always made me leave his office with a new solution to try and a smile on my face. I am indebted to him more than he knows.

I am thankful to my co-supervisor, Dr. Armaghan Salehian, for her constant assistance and academic guidance throughout the development of this thesis. Without her support this thesis would not have been completed. My experience working with her has been nothing short of amazing.

Dr. Derek Wright and Dr. Hiren Patel deserve all the thanks I can give them for their willingness to push their own work aside to read and revise my thesis. Their many helpful suggestions made this thesis more coherent and much stronger.

To my colleague and collaborator in this project, Egon Fernandes, who gave me enormous technical support and hands-on help in many aspects of this project. I enjoyed our insightful conversations over our countless coffee breaks that led to the development of many ideas in this thesis.

To my parents and my sisters, for all their unfailing emotional support throughout this journey. And to my friends who have given me all their love and encouragement. My time in Waterloo was made memorable because of the many friends who became a part of my life. Catherine, Kirk, Ian, Colin, Jennifer, Jacqueline, Sarah, Luke, Amir, Ramin, Anna, and my backpacking buddy Adel, for our memorable trip into the mountains and to many other friends whom I haven't named here. 


\section{Dedication}

Dedicated to my grandmother Maryam. 


\section{Table of Contents}

$\begin{array}{ll}\text { List of Tables } & \text { ix }\end{array}$

$\begin{array}{ll}\text { List of Figures } & \mathbf{x} \\ \end{array}$

1 Introduction $\quad 1$

1.1 Self-Contained Wireless Sensor Nodes ........................................................................ 1

1.1.1 Electromagnetic Energy Harvesting .............................................................................. 3

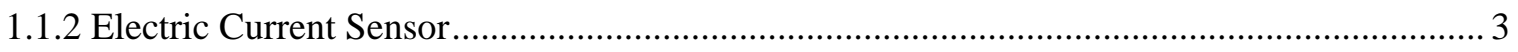

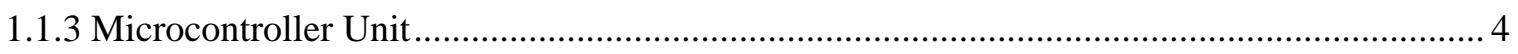

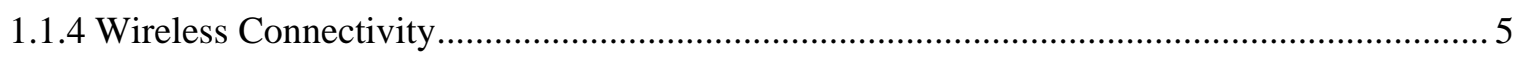

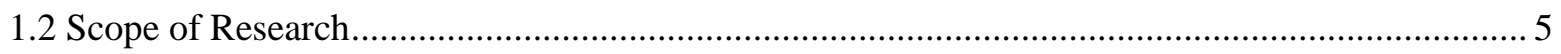

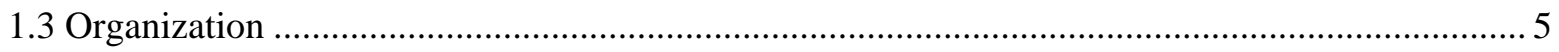

2 Self-Contained Sensor Nodes $\quad 6$

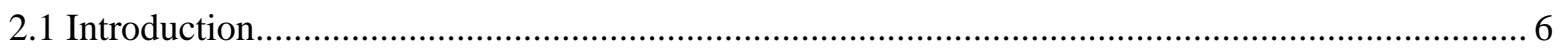

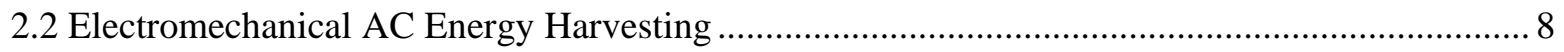

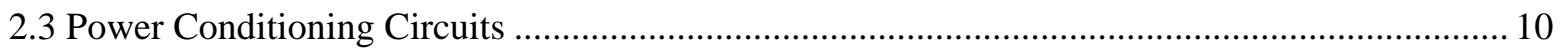

2.3.1 Available Power Conditioning Technologies ...................................................................... 11

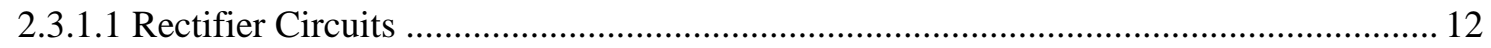

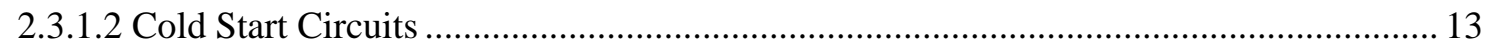

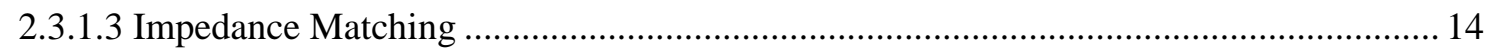

2.3.1.4 Previous work in literature on Power Conditioning Circuits....................................... 15

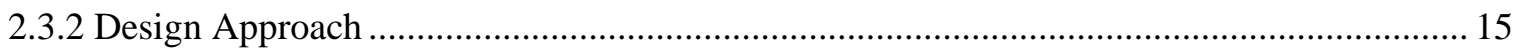

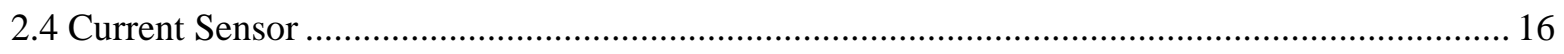

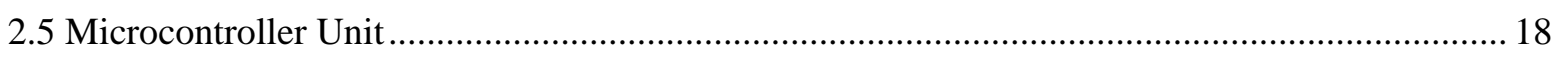

2.5.1 Microcontroller Power Consumption and System Life Time............................................ 18 


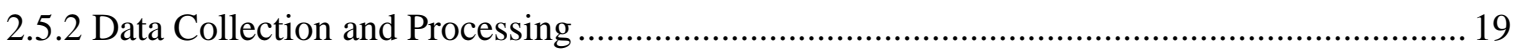

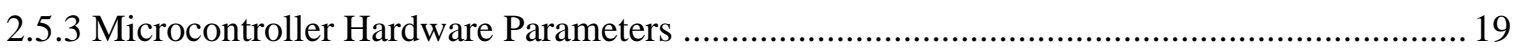

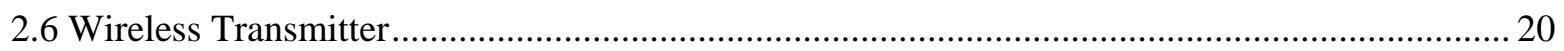

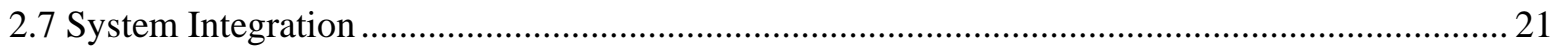

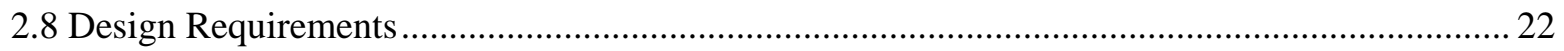

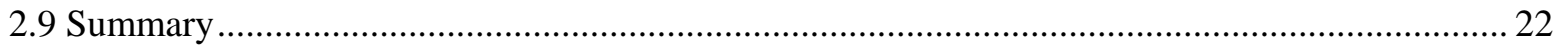

3 Design Approach $\quad 23$

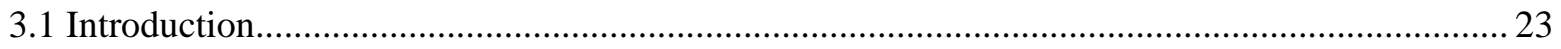

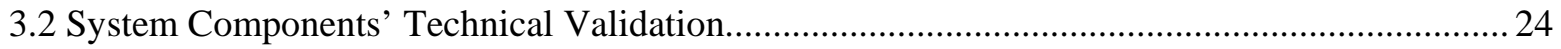

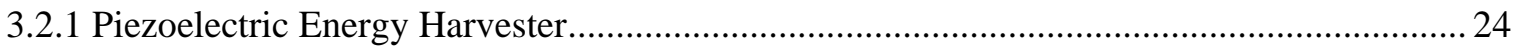

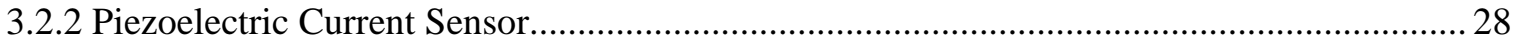

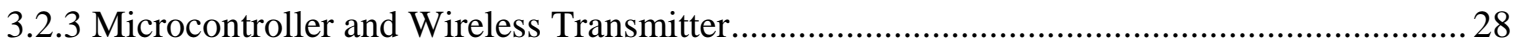

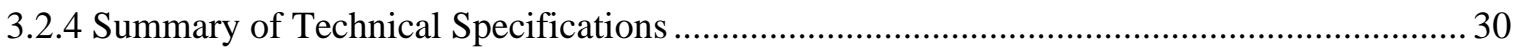

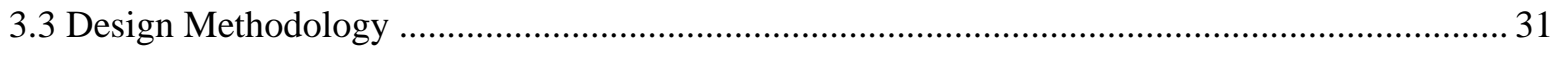

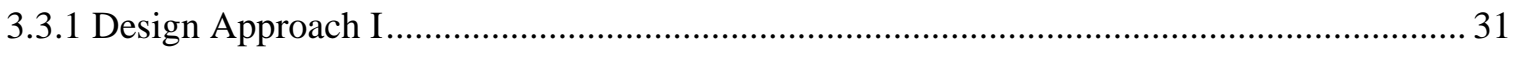

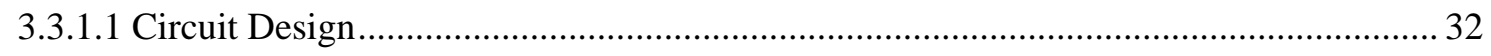

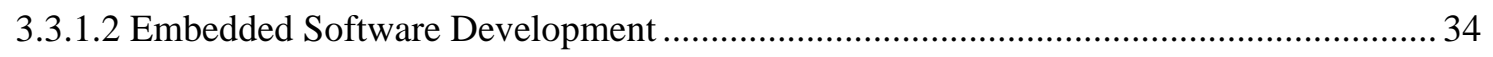

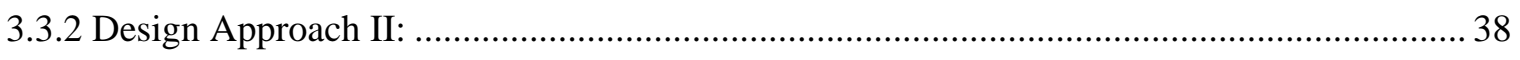

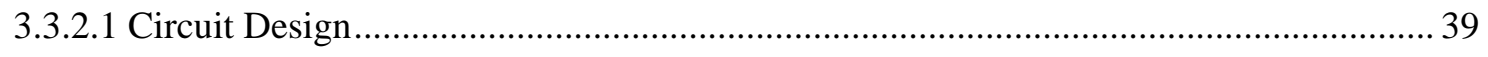

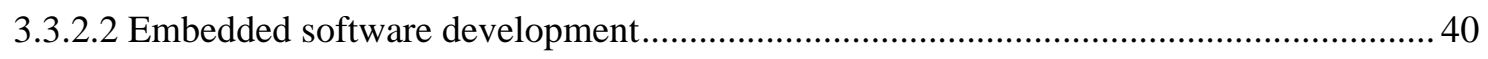

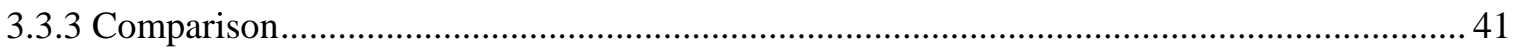

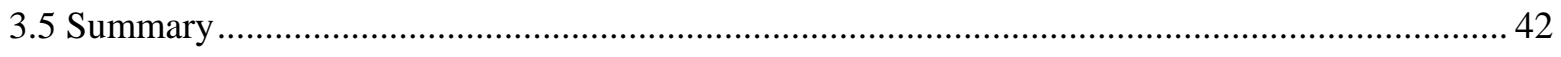

4 Implementation and Testing $\quad 43$

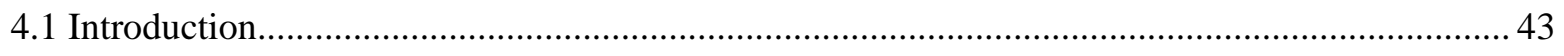

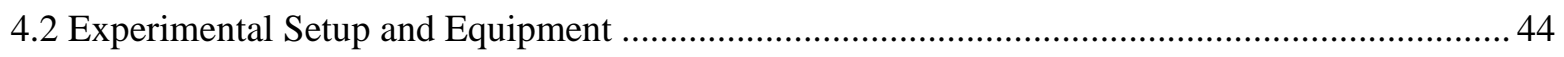




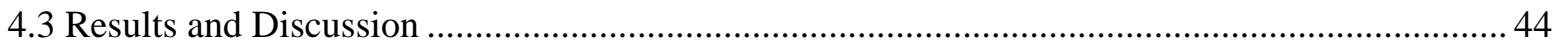

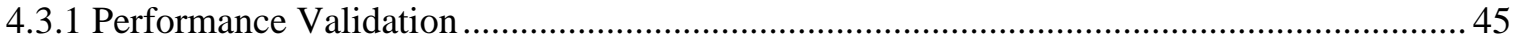

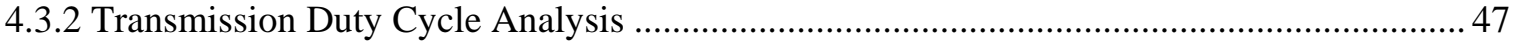

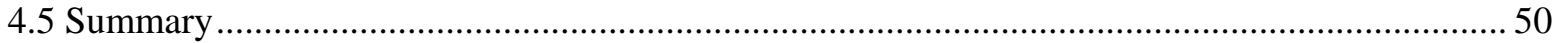

5 Conclusions \& Future Work $\quad 51$

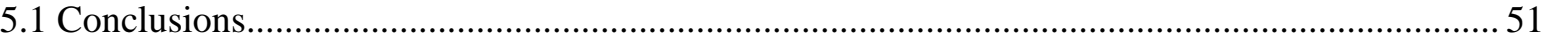

5.2 Future Work

6 References $\quad 54$ 


\section{List of Tables}

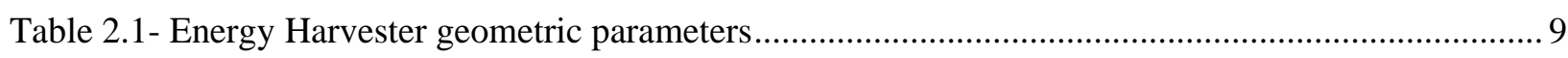

Table 2.2- Design specifications of the piezoelectric AC current sensor designed at the UW Energy

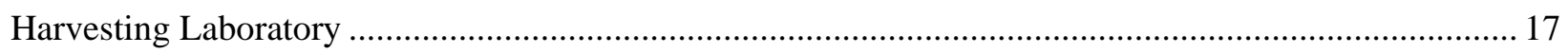

Table 3.1- Feature Analysis ATMEGA1281R212 vs. CC2640R2F [39] [40]....................................... 28

Table 3.2- Operational condition of the CC2640R2F Wireless MCU [39] ............................................. 30

Table 3.3- CC2640R2F’s power modes and power management features [39] ....................................... 37

Table 4.1-Transmitted ADC values for two arbitrary signal inputs to the wireless sensor node................ 46

Table 4.2- Duty Cycle of operation as a function of current passing through the wire ............................ 48 


\section{List of Figures}

Figure 1.1- Overall schematic of a self-powered wireless sensor node ................................................. 2

Figure 1.2- Schematic of an electromagnetic energy scavenging unit................................................ 3

Figure 1.3- Schematic of the miniature AC current sensor [4] ........................................................... 4

Figure 2.1- Operational sequence of an integrated wireless sensor node ............................................... 7

Figure 2.2- Piezoelectric Energy Harvester operation mechanism [9] …............................................... 8

Figure 2.3- (a) Piezoelectric bimorph cantilever model (b) EH assembly ............................................. 9

Figure 2.4- (a) Schematic of the harvester (mm), (b) Fabricated PZT EH ............................................ 10

Figure 2.5- Piezoelectric electromechanical model with a resistive load .............................................. 11

Figure 2.6- Full-wave rectifier topologies: (a) diode rectifier (b) diode-tied MOS rectifier (c) gate crosscoupled NMOS rectifier (d) cross-coupled rectifier ........................................................................... 12

Figure 2.7- Active rectifier with cross-coupled PMOS switches [16] ................................................... 13

Figure 2.8- Voltage across the storage unit with and without cold start circuit....................................... 13

Figure 2.9- Power conditioning circuit's flow diagram [24] ............................................................ 14

Figure 2.10- The power conditioning circuit integrated with a bimorph piezoelectric generator............... 15

Figure 2.11- Piezoelectric AC current sensor [27] ........................................................................... 16

Figure 2.12- Sensor's output (RMS voltage) as a function of the current passing through the wire. Different

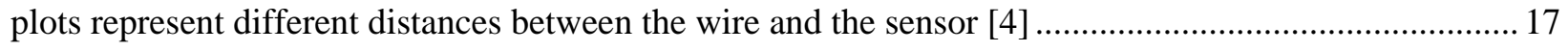

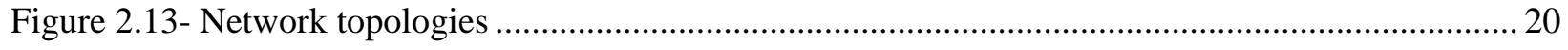

Figure 3.1- (a) A photograph of the electromechanical piezo-electric energy-harvesting unit. (b) Frequency Response Function (FRF) of the PZT EH with peak voltage at the resonance frequency of $60.2 \mathrm{~Hz}$........ 25

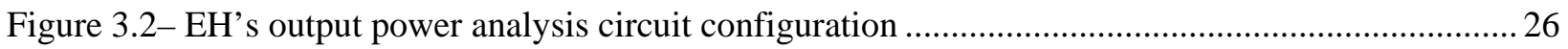


Figure 3.3- Output power of the EH unit measured by the load-sweep of the EH mounted on a wire carrying different electric current values.

Figure 3.4- EH's RMS power output measurement at its optimum load and as a function of the current passing through the wire

Figure 3.5- Functional block diagram of the CC2640R2F Wireless MCU

Figure 3.6- 48-pin CC2640R2F Wireless MCU package $(7.00 \mathrm{~mm} \times 7.00 \mathrm{~mm})$ 30

Figure 3.7- Design I architecture 32

Figure 3.8- Design I circuit schematic. 33

Figure 3.9- The charging behavior of the $1000 \mu \mathrm{F}$ capacitor under different input conditions. 34

Figure 3.10-Wireless MCU process flow chart 35

Figure 3.11- ADC sampling procedure 36

Figure 3.12- Design II architecture 38

Figure 3.13-Supply voltage configuration for internal DC-DC regulator. 39

Figure 3.14- Design II circuit schematic..... 40

Figure 3.15- Wireless MCU process flow chart 41

Figure 4.1 - Sensor output signal and the transmitted ADC values .45

Figure 4.2- Charging/Discharging behavior of the Storage Unit as data packets are transmitted from the sensor node when the wire is conducting a current of 7.6A. .48

Figure 4.3- Charging/Discharging behavior of the Storage Unit as data packets are transmitted from the sensor node when the wire is conducting a current of (a) 11.8A, (b) 19.4A, (c) 25.7A . .49

Figure 4.4- Voltage Detector and the Trigger Stabilizer. .49 


\section{Chapter 1}

\section{Introduction}

\subsection{Self-Contained Wireless Sensor Nodes}

The growing concern over enhancing the energy consumption efficiency within residential and commercial buildings has led to the recent research efforts in the development of intelligent power management systems. Achieving the targeted environmental and economic objectives requires an automated and intelligent infrastructure capable of monitoring the energy usage by individual appliances within a building. Such intelligent automatization involves the deployment of advanced metering, control, and communication devices that could transform the outdated electric distribution network within residential and commercial settings to a smart and intelligent system capable of providing real time information that can be processed and used to monitor and enhance the power distribution efficiency within buildings.

Motivated by this vision, new technologies have been developed that enable users to monitor energy consumption by electric appliances within a building. Among these technologies, Wireless Sensor 
Networks (WSNs) have received an increased attention due to their promising approach in forming an intelligent power management system. WSNs consist of low-powered, non-invasive and cost-effective intelligent sensor nodes that are capable of gathering, analyzing, and transmitting the sensory information to a central hub for post-processing purposes.

Although wireless sensor nodes are designed and programmed to operate with minimal power consumption, their operational lifetime is often limited by the node's battery lifespan. Therefore, having a self-sustained system where the power required for the device's operation is harvested from the ambient energy in the environment eliminates the need for batteries and therefore prolongs the life and durability of sensor nodes [1].

As shown in Figure 1.1, a self-contained wireless sensor node is an integrated solution that includes an Energy Harvester (EH), an electric current sensor, a power conditioning circuit, a control unit, and a wireless communication device. Each component plays an indispensable role in the operation of the device. Therefore, to provide the reader with the background information necessary to realize the joined and interconnected operation of a wireless sensor node, the function and role of each block and its respective components will be introduced independently in this chapter.

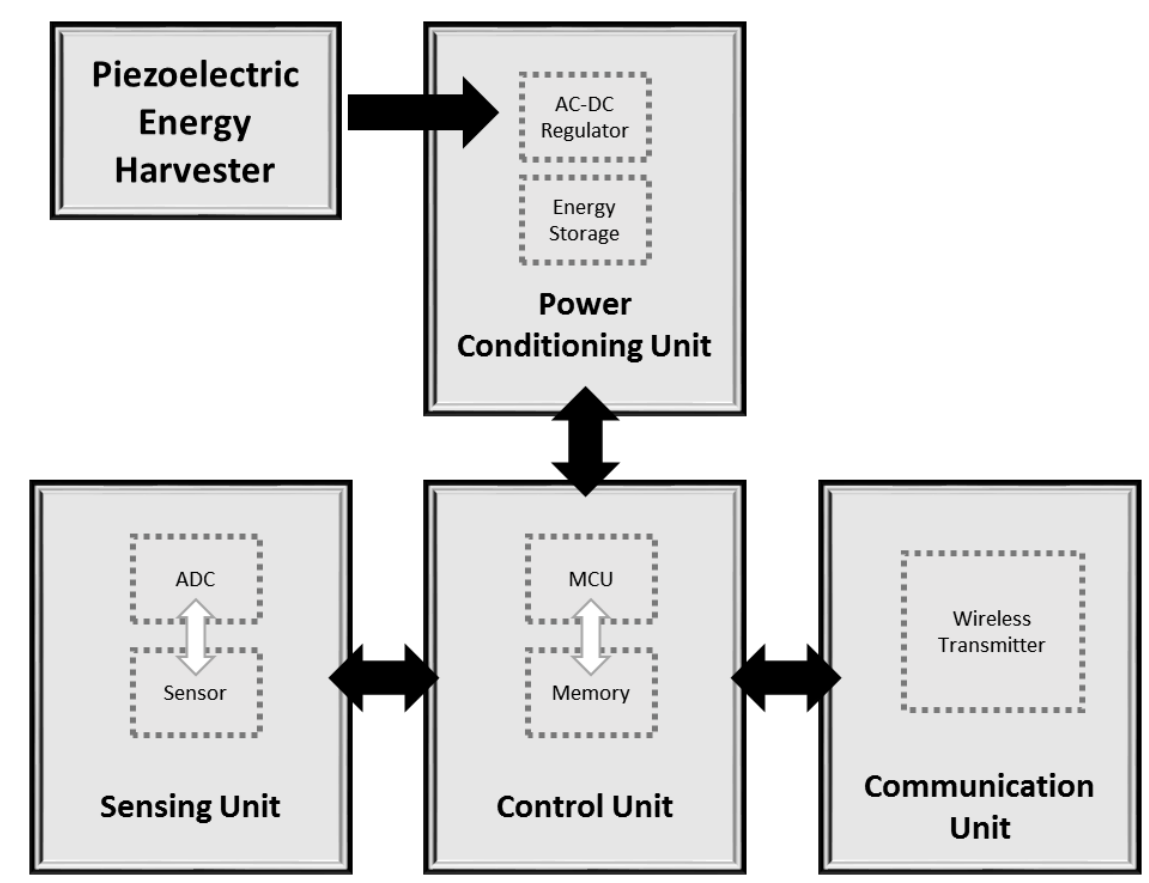

Figure 1.1- Overall schematic of a self-powered wireless sensor node 


\subsubsection{Electromagnetic Energy Harvesting}

Electromagnetic energy harvesting is an attractive method for scavenging the ambient energy from the surroundings of an electric wire. The main purpose of energy harvesting in this case is to provide an alternative power source which would ultimately eliminate the need for batteries resulting in a prolonged operation of sensor nodes. Figure 1.2 shows the schematic of an electromagnetic EH and its placement orientation. The EH will be placed on a cord connecting a given home appliance to the electric receptacle. As the result of the Alternating Current (AC) passing through the wire, an electromagnetic field is generated around it. The intensity of this electromagnetic field depends on the power consumed by the appliance. Through the conversion of this ambient electromagnetic energy into the mechanical vibrational energy and subsequently to electrical energy, energy is non-invasively harvested from the wire.

One of the most common configurations for piezoelectric electromagnetic energy harvesting is the cantilever beam structure [2]. The electromagnetic field created by the AC current transduces a force on a permanent magnet placed in the vicinity of the wire. By placing this magnet at the end of a cantilever beam, the force applied to the magnet can create a mechanical vibrational force along the beam. The exhibited mechanical vibrational energy by the oscillating beam can be transferred to electrical charge through exploiting the unique characteristics of Piezoelectric (PZT) materials [3]. The generated electric charge will be stored in a storage unit (a rechargeable battery, super-capacitor, etc.) and will be used by the wireless sensor node as needed.

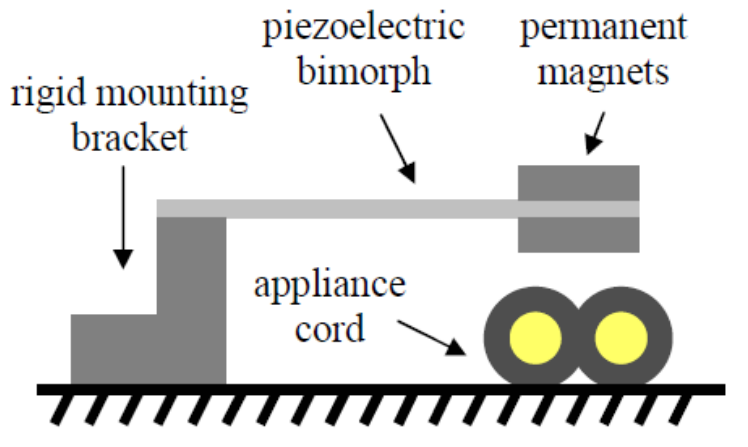

Figure 1.2- Schematic of an electromagnetic energy scavenging unit

\subsubsection{Electric Current Sensor}

The current sensor is responsible for monitoring the intensity of the AC current passing through the wire. A non-contact measurement method allows for the integrated device to be installed with minimal effort and without any service disruptions. A number of different technologies and devices have been 
developed that enable users to obtain these measurement in a non-invasive manner. Non-contact sensor technologies such as the Rogowski coils or Hall sensors are not suitable for the purpose of our application due to their size and external power requirements [2]. The sensor used in this project had been developed previously by the University of Waterloo's Energy Harvesting team and was tested and verified at the Ottawa Hydro's testing facility [4]. The sensor's operation is similar to that of the Energy Harvester (Figure 1.3 [4]). The force transduced on the permanent magnet at the tip of a piezoelectric cantilever (as the result of the electromagnetic field generated around the wire), produces an AC signal that is measured and calibrated to the amplitude of current passing through the wire.

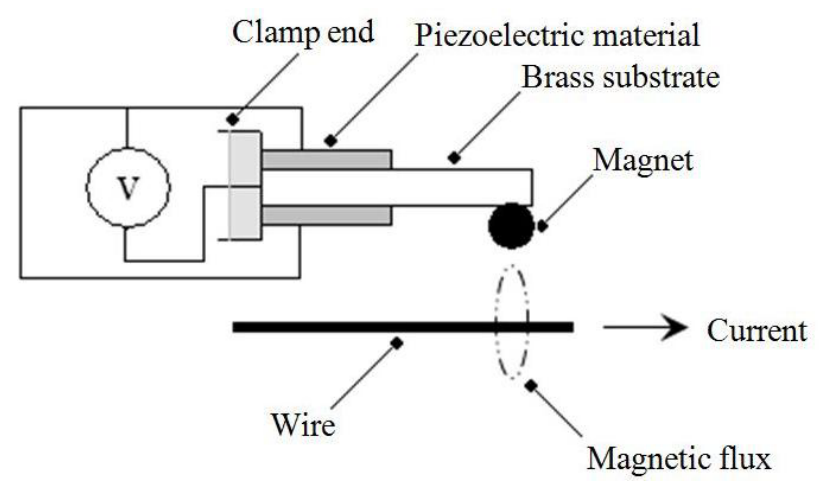

Figure 1.3- Schematic of the miniature AC current sensor [4]

\subsubsection{Microcontroller Unit}

The Microcontroller Unit (MCU) provides the platform for localized analysis of the sensor's output. The collected data from embedded processing of this signal will be transmitted through the selected digital communication protocol. Since the energy collected from the proximity of the wire by the EH is considerably small (in the microwatts range), the MCU should be able to operate under a very limited power budget. Selecting a suitable Microcontroller requires a careful assessment of MCU's parameters as well as the end use application. The complex self-contained wireless sensor node requires a Microcontroller with enhanced accuracy and reliability in collecting the analog signal. Units that are commonly used in WSN applications are relatively small in size and entail an ultra-low power profile. 


\subsubsection{Wireless Connectivity}

With the different wireless technologies available, finding the right wireless platform to meet the specific requirements of the given application could become cumbersome. In the case of the sensor node for electricity monitoring applications, considerations such as data throughput, range, power consumption, cost, and ease of integration should be used to determine the most suitable technology. There are other considerations such as network security and network topology that go beyond the scope of this project and will not be discussed in this report. Once the frequency band and the appropriate wireless platform are selected, the RF transceiver is programmed and integrated with the rest of the system in order to transmit data packets obtained by the MCU to the central connectivity hub.

\subsection{Scope of Research}

This work seeks to report on the design, development, and integration of a self-contained and non-invasive integrated system intended for real-time monitoring of electricity usage within residential and commercial buildings. The design framework introduced here provides a systematic approach that can be easily extended to other similar applications, enabling users to develop a customized system. Furthermore, it provides a framework and guideline for the development of a system that enables the Microcontroller and the Radio Frequency (RF) devices to read and transmit the data gathered from an external sensor device.

\subsection{Organization}

This thesis is divided up into three main sections. In Chapter 2, an overview of technologies available in the market to the end users as well as the emerging technologies within the research community are presented to the readers. Each system is broken down into its building blocks with a thorough examination of their components. Chapter 2 is concluded by the extraction and analysis of operational conditions of a wireless sensor node to drive a set of system requirements. In Chapter 3, several different design approaches are investigated and examined. These designs are compared based on their merits, advantages, and disadvantages and the most suitable design is selected for implementation and testing. In Chapter 4, details regarding the testing of the selected architecture is presented. Lastly, the unit's functionality within the tolerance of the design requirements is demonstrated to verify the presented design. 


\section{Chapter 2}

\section{Self-Contained Sensor Nodes}

\subsection{Introduction}

In order to actively monitor individual components of a system, an intelligent network of sensor nodes is required to gather, process, and transmit the sensory information according to the application requirements. These intelligent sensor nodes, provide real time information regarding the physical or environmental conditions of the system under study, such as temperature, pressure, motion, etc. The network of interconnected sensor nodes construct a Wireless Sensor Network (WSN) where the sensory information acquired by each node is processed and transmitted to a central information hub. In the case of the WSN designed for electricity monitoring applications, the data gathered from sensor nodes provide the user with an overall view of energy distribution within a building. Further processing of the acquired data can offer key and essential information regarding the energy consumed by individual appliances within the building. 
However, design and development of a single wireless sensor node is hindered by some of its inherent limitations such as its power consumption, data storage capacity, data processing speed and wireless communication bandwidth [5]. The objective of this research project is to tackle some of these constraints to develop and design a self-powered wireless sensor node that can continuously monitor the power consumption of electric appliances within residential and commercial buildings. Throughout this chapter, we will thoroughly investigate a number of systems developed by other research groups working on this topic. Furthermore, we will have an overview of the end-solutions available to the users. These selected systems are broken down to their building blocks as they are examined based on their merits and shortcomings.

Due to the defined scope of this project, less emphasis has been placed on the design of the EH. An electromagnetic EH fabricated by the Energy Harvesting team at the University of Waterloo was used as a prototype for the design of the interface circuitry as well as the control and communication systems. We will be integrating the circuitry developed here with the meandering PZT EH that is under development by the University of Waterloo research team and has been reported in literature [6]. However, a brief and preliminary review of EHs will be done in the following section to provide readers with the necessary information regarding the operation of EHs and their electromechanical characteristics.

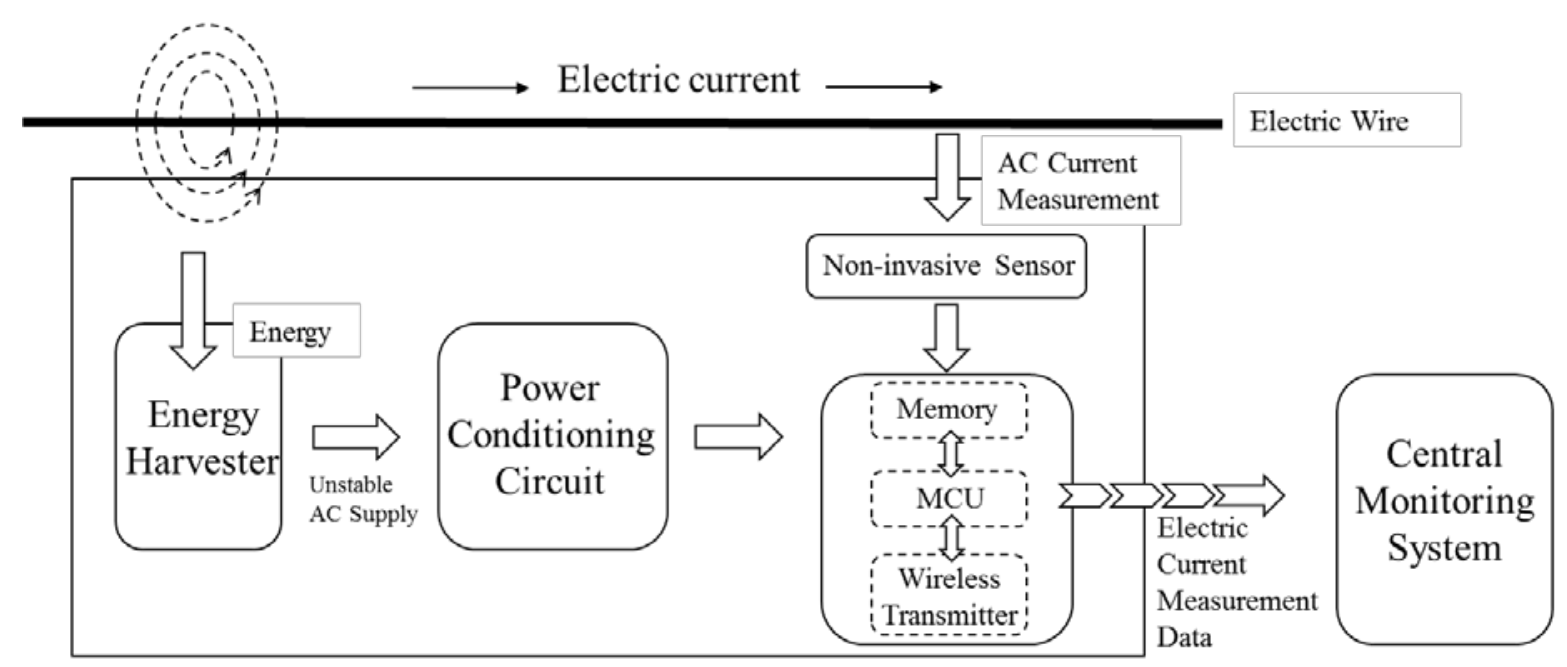

Figure 2.1- Operational sequence of an integrated wireless sensor node 


\subsection{Electromechanical AC Energy Harvesting}

Energy scavenging from the environment has received much attention in the past decade. The reduced power requirements of electronic devices has led to an extensive research in this area with the ultimate goal of powering small electronics using the harvested energy from the environment to eliminate the need for external power sources such as batteries. Depending on the end use application of the electronic device under consideration, the nature of ambient energy source could vary. Vibration, light, and thermal gradients have all been shown to be promising sources for energy harvesting [2].

In the case of self-contained and non-invasive wireless sensor nodes for electricity monitoring applications, the electromagnetic energy is the dominant and most accessible source of ambient energy available to the EH [7]. Therefore, electromechanical AC energy harvesting is considered to be the most promising approach in powering the electronics and eliminating the need for periodic battery replacement in such sensor nodes.

An electromechanical PZT EH typically consists of a cantilever beam with one or two piezoceramic layers attached to it (Figure 2.2). A permanent magnet will couple to the magnetic field generated by the alternating current flowing through the nearby wire [8]. As a result, the attached magnet will be excited by the force induced by the magnetic field generated from the current flowing through the wire. The mechanical vibrational energy from the oscillating magnet can be transferred to electrical energy using the piezoelectric effect. Piezoelectric materials will accumulate electric charge in response to the applied mechanical stress. Therefore, an electric potential will be generated as the cantilever beam deforms under vibrational stress. By matching the resonant frequency of the EH with that of the alternating magnetic field, the beam will sustain maximum displacement leading to a maximized output power [7].

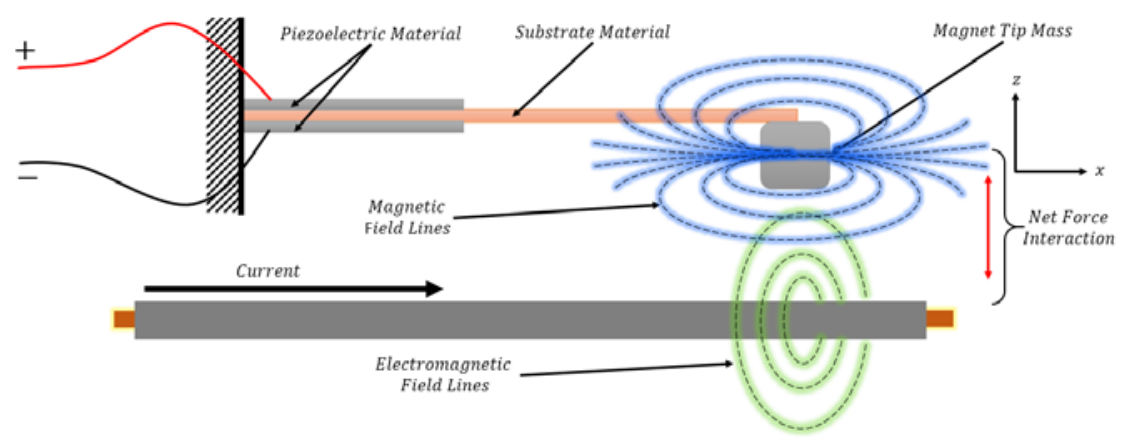

Figure 2.2- Piezoelectric Energy Harvester operation mechanism [9] 
The average power output from an EH is dependent on parameters such as its size, geometry, the input stimulus and frequency, the environment of operation, and the impedance of the interface circuitry responsible for energy regulation and storage [10]. This power output should be able to fulfil the power requirements of electronic devices within the integrated system. Therefore, it is important to conduct a preliminary investigation of power output characteristics of the EH and also the power consumption profile of integrated electronics.

As specified previously, since the focus of this research project has been placed mainly on the design and development of a flexible interface circuitry and also the integration of microcontroller and the wireless connectivity components, less emphasis was put on the design of the energy harvesting unit. Therefore, a PZT EH unit previously developed by the University of Waterloo's Energy Harvesting team was used throughout this project. This unit is a simple piezoelectric bimorph cantilever capable of harvesting energy from the electromagnetic field of a $60 \mathrm{~Hz}$ electric current passing through a wire. The specific design parameters of this energy harvester are provided in Table 2.1 [9].

Table 2.1- Energy Harvester geometric parameters

\begin{tabular}{|c|c|c|c|}
\hline Geometric Parameter & Name & Units & EH \\
\hline Substrate Length & $L_{\text {eff }}$ & $\mathrm{mm}$ & 27.36 \\
\hline PZT Length & $L_{\tilde{p}}$ & $\mathrm{~mm}$ & 10.75 \\
\hline PZT Gap Length & $L_{1}$ & $\mathrm{~mm}$ & 1 \\
\hline PZT End Length & $L_{2}$ & $\mathrm{~mm}$ & 11.75 \\
\hline Width & $w$ & $\mathrm{~mm}$ & 2.86 \\
\hline PZT Thickness & $t_{\tilde{p}}$ & $\mathrm{~mm}$ & 0.127 \\
\hline Substrate Thickness & $t_{\tilde{s}}$ & $\mathrm{~mm}$ & 0.254 \\
\hline Magnet Length & $L_{m}$ & $\mathrm{~mm}$ & 3.175 \\
\hline Magnet Thickness & $t_{m}$ & $\mathrm{~mm}$ & 3.175 \\
\hline Magnet Width & $w_{m}$ & $\mathrm{~mm}$ & 12.65 \\
\hline
\end{tabular}

Figure 2.3 [9] shows the EH architecture as well as its assembly. The unit is less than $30 \mathrm{~mm}$ in length, includes $10 \mathrm{~mm}$ of piezoelectric material, and sustains a total volume of $154 \times 10^{-12} \mathrm{~cm}^{3}$.
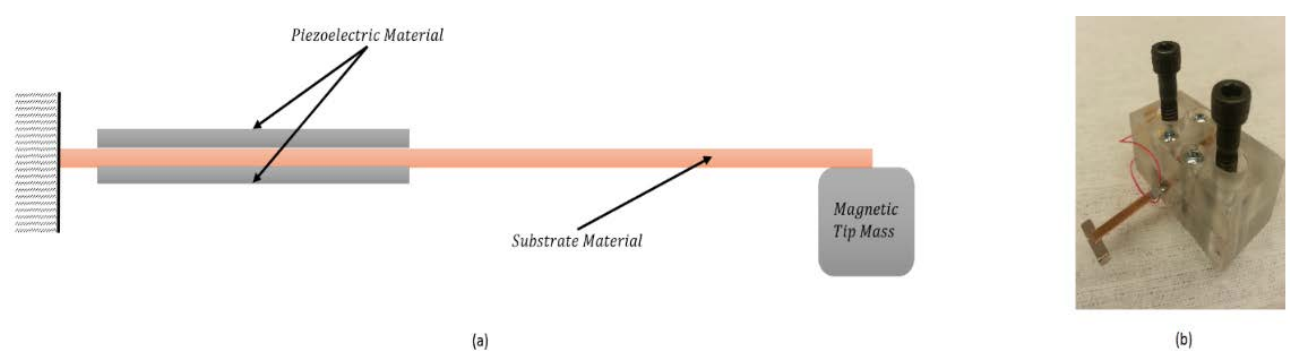

(b)

Figure 2.3- (a) Piezoelectric bimorph cantilever model (b) EH assembly 
The circuitry developed here will be ultimately integrated with a more advanced PZT EH that is developed and reported by our research team [6]. Compared to the simple cantilever structure of similar area, the newly developed design has the required fundamental natural frequency of $60 \mathrm{~Hz}$ and maintains a high power output. This centrally supported meandering geometry (presented in Figure 2.4 [11]) uses the symmetry around a clamped beam to mirror two zigzag structures which further reduce the overall design area.

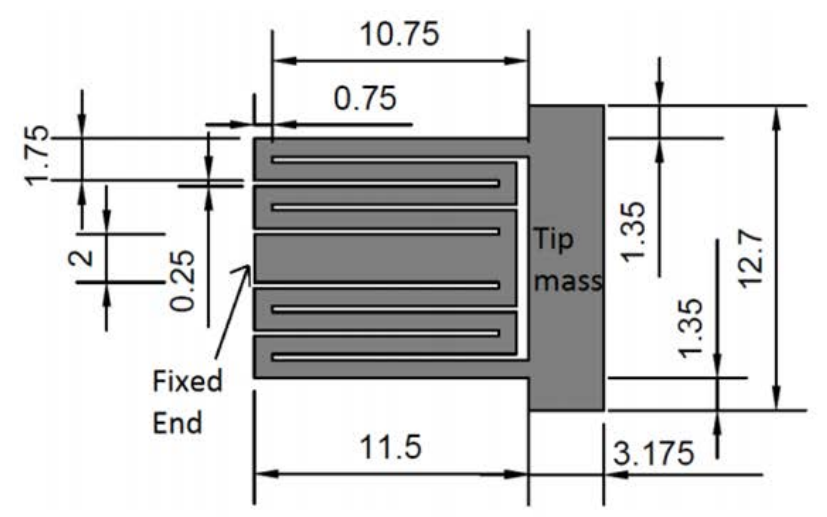

(a)

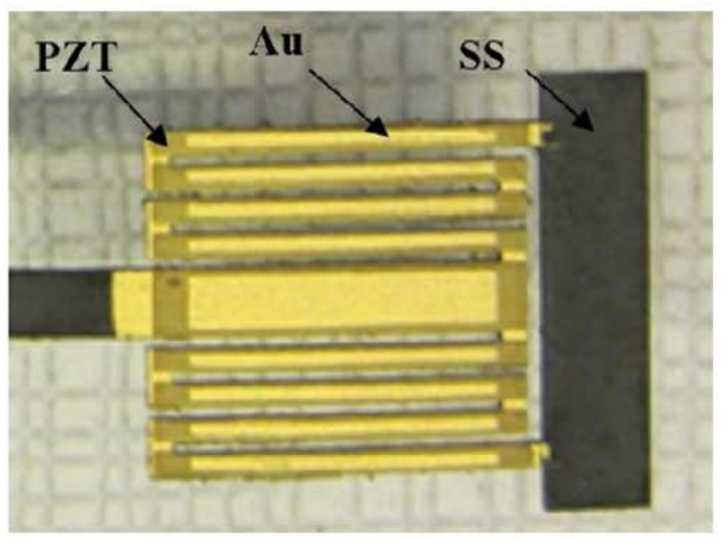

(b)

Figure 2.4- (a) Schematic of the harvester (mm), (b) Fabricated PZT EH

\subsection{Power Conditioning Circuits}

The piezoelectric EH can be modeled by a sinusoidal current source (representing the vibrating beam) in parallel with its internal electrode capacitance (Figure 2.5) [12]. The vibration of the piezoelectric beam generates an AC output. The amplitude of this current depends on the degree of beam deflection caused by the vibrational excitement. An accurate prediction of the output power for a given vibrational excitement requires a more sophisticated circuit model. The behaviour of a PZT EH can slightly differ from the simplified electromechanical equivalent circuit presented in Figure 2.5. The resonance frequency shift and the piezoelectric voltage change lead to the variation of the EH's internal impedance. However, this simplified model is sufficient for the design of the interface circuitry and will be used in this work.

The AC voltage across the load connected to the EH needs to be rectified, regulated, and conditioned before it can be used by the load circuitry. The rectified current can charge an energy storage unit such as a battery or a supercapacitor which can be subsequently regulated and conditioned to power the integrated electronic devices. 


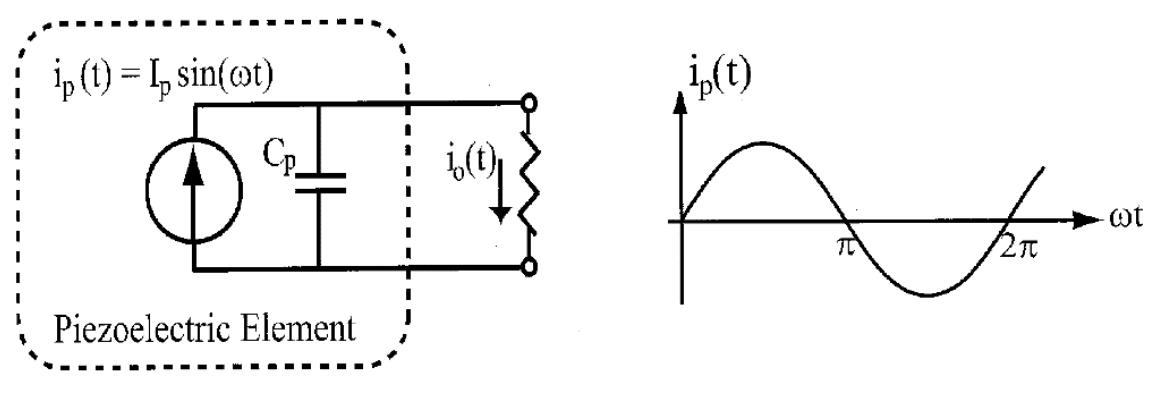

Figure 2.5- Piezoelectric electromechanical model with a resistive load

The use of PZT EH as a power source is constrained by some of its inherent characteristics. The EH maintains a high internal impedance that limits the amount of current that can be directly supplied to the subsequent circuitry. Most EHs are only able to provide an output power in the range of $10 \mu \mathrm{W}$ to $100 \mu \mathrm{W}$ which puts a constraint on the energy available to operate the control and communication devices [13]. Furthermore, the low output voltage of the PZT EHs sets yet another constraint on the development of an efficient interface circuitry between the harvester and the integrated electronic devices. Considering the above restrictions and challenges, it is advantageous to develop an application specific architecture where the specifications of the energy harvesting unit and the integrated electronic devices are taken into careful consideration during the design process. In order to provide the reader with some of the available power conditioning technologies, a number of designs will be reviewed in the following section.

\subsubsection{Available Power Conditioning Technologies}

Interface circuitries ranging from simple bridge rectifiers to active converter circuits with intelligent control systems have been reported in the literature for power conditioning within energy harvesting systems. Due to the limitations associated with the operation of EH units, the focus of most works have been placed on maximizing the system's efficiency according to the requirements of the end use application [14]. Power conditioning technologies differ in their complexity and efficiency and they are designed for operation within a unique environment. Therefore, although some circuit blocks may be used across applications, most power conditioning circuits are uniquely optimized [15]. A number of technologies reported in the literature that are frequently used for power conditioning within energy harvesting systems will be presented here. 


\subsubsection{Rectifier Circuits}

As pointed out previously, PZT EHs generate a time-varying AC output. For a given EH, the amplitude of the output voltage varies according to the environmental settings and operating conditions. Factors such as temperature, the distance between the $\mathrm{EH}$ and the wire, frequency and amplitude of the current passing through the wire can all influence the output voltage retrieved from the EH. Therefore, in order to make this voltage usable by other electronic devices, voltage rectification and regulation is necessary.

Full-bridge rectifiers, consisting of diodes or transistor switches, are the most commonly reported circuits in the field of energy harvesting [16]. In the case of diode rectifiers, the forward bias voltage drop could further reduce the maximum output voltage available to the load circuitry. To alleviate this problem, Schottky diodes have been used to reduce this forward voltage drop. In spite of that, the current leakage of diodes in their reverse bias still accounts for power loss and reduced efficiency (Schottky diodes in particular are known for their increased reverse current leakage). Some of the simplest yet most commonly used full-wave rectifier topologies are shown in Figure 2.6 [16].

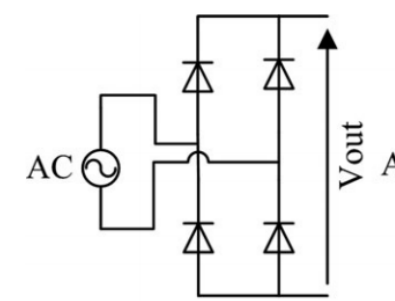

(a)

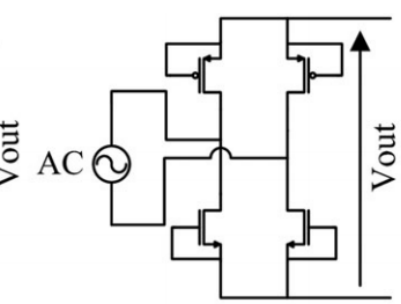

(b)

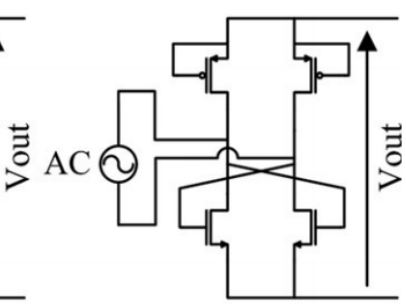

(c)

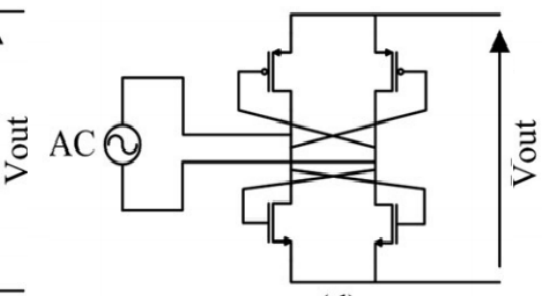

(d)

Figure 2.6- Full-wave rectifier topologies: (a) diode rectifier (b) diode-tied MOS rectifier (c) gate cross-coupled NMOS rectifier (d) cross-coupled rectifier

As shown in Figure 2.6, diodes can be replaced by MOSFETs to further enhance the rectification efficiency. Nevertheless, the threshold voltage of transistors can still have an impact on the system's efficiency. Despite reducing the voltage drop, having a high input voltage (greater than $1.5 \mathrm{~V}$ in most cases) is a prerequisite for the use of these architectures in order for transistors to completely switch between ON and OFF modes during the rectification process [17]. Such high output voltage is commonly unattainable by most EHs.

Active or synchronous rectifiers take on a different approach in reducing the voltage drop across MOSFETs. In this case, the control circuitry (mainly the comparator) is responsible for detecting the 
amplitude of the output AC voltage from the EH. Using the output signal from the comparator, transistors will open at precise instances to allow voltage rectification to take place. However, the addition of voltage comparator to the design places an extra burden on the EH due to the additional power consumed by these units [18]. This additional power consumption is undesirable since the overall efficiency of the system can be dramatically impacted by it.

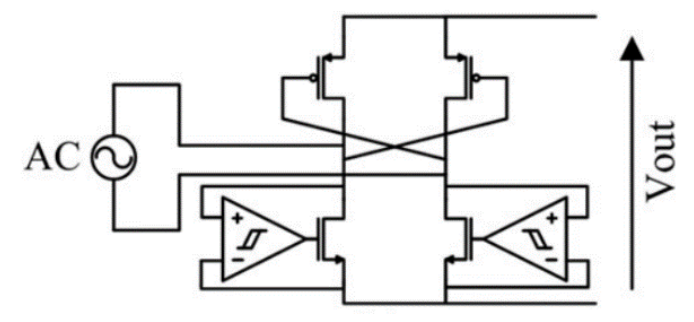

Figure 2.7- Active rectifier with cross-coupled PMOS switches [16]

\subsubsection{Cold Start Circuits}

Due to the surge current drawn by the power conditioning circuit, as well as the Microcontroller unit, the voltage across the storage capacitor cannot exceeds a nominal voltage (typically lower than $1 \mathrm{~V}$ ) leading to a cold start of the circuit and a collapse of the integrated system (Figure 2.8) [19]. Different methodologies have been developed to address this issue. Through isolating the active components of the circuit using CMOS switches, the electric current delivered from the storage unit to the integrated electronic devices can be blocked until a certain minimum voltage across the storage capacitor is reached [20]. Using a different approach, an integrating control block is implemented as the power management unit. In this case, the power management unit will enable the load circuitry only after the voltage across the storage unit surpasses the minimum required voltage needed to overcome the cold start of the circuit [21].

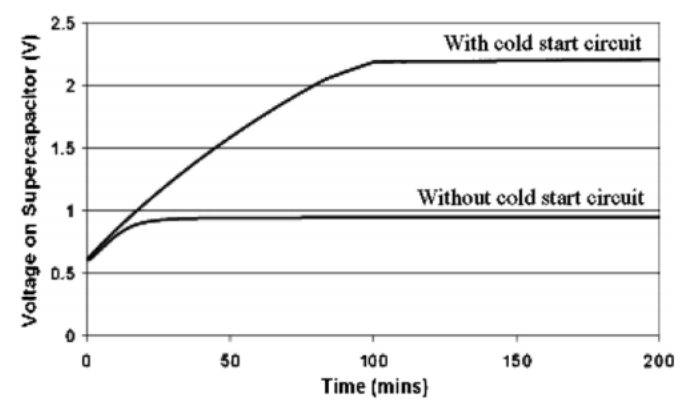

Figure 2.8- Voltage across the storage unit with and without cold start circuit 
Figure 2.9 [24] shows the block diagram for the operation of a cold-start circuit. A low-powered voltage detector will be responsible for monitoring the voltage across the storage unit. Once the minimum required voltage is achieved, an N-channel MOSFET switch will reintroduce the voltage regulator to the circuit.

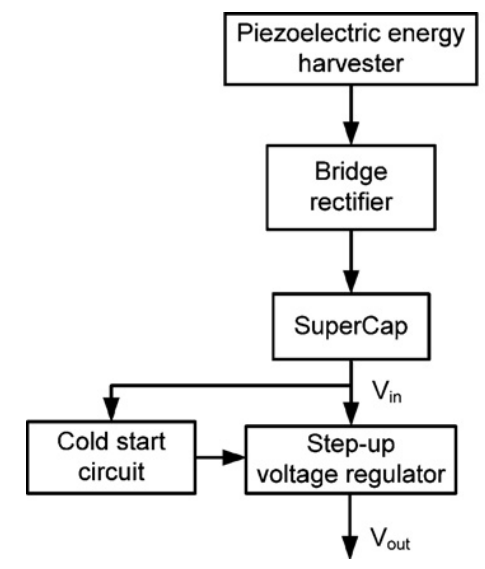

Figure 2.9- Power conditioning circuit's flow diagram [24]

\subsubsection{Impedance Matching}

As it was pointed out earlier in the text, the maximum power output obtained from the EH is affected by operational factors and design considerations. Given a particular EH, the maximum power output derived from the harvester can be determined through an output power analysis under a load sweep. The load sweep (also known as the impedance matching technique) can identify the optimum electrical load capable of maximizing the power output from an isolated EH. This power output will be used in calculating the nominal efficiency of the system for power transfer from the EH to the MCU by the power conditioning circuit.

In order to obtain the maximum power delivered from an energy harvesting source, the impedance of the power conditioning circuit should match that of the EH [22]. A further analysis has indicated that the maximum voltage output of the piezoelectric EH is clamped by the voltage across the storage capacitor [23]. Therefore, with effective impedance matching and also through accurate modeling of the EH unit and determining a reservoir capacitor with a suitable storage capacity, an optimal power conditioning circuit can be designed. 


\subsubsection{Previous work in literature on Power Conditioning Circuits}

An example of power conditioning circuit schematic that is powered by a bimorph piezoelectric EH generator is shown in Figure 2.10 [24]. The EH in this case operates at the frequency of $67 \mathrm{~Hz}$ and can yield an RMS output power of $240 \mu \mathrm{W}$. In order to supply a stable output voltage of $3.3 \mathrm{~V}$ to the MCU, a stepup voltage regulator has been included to boost the voltage across the supercapacitor into a regulated and constant output voltage of $3.3 \mathrm{~V}$. The problem associated with the cold start of the circuit caused by the current leakage through the regulator is overcome by introducing a "cold-start circuit" that temporarily disconnects the voltage regulator from the circuit until the minimum voltage needed to overcome the cold start is achieved.

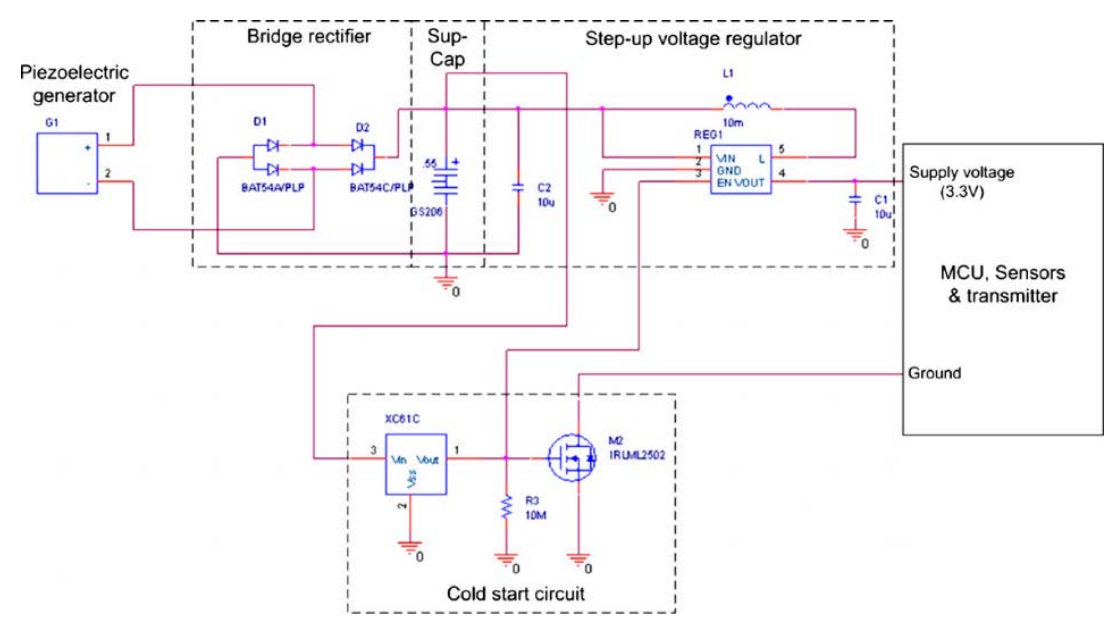

Figure 2.10- The power conditioning circuit integrated with a bimorph piezoelectric generator

\subsubsection{Design Approach}

A practical interface circuitry must be compatible with both the $\mathrm{EH}$ and the control and communication devices while being operational over a wide range of operating conditions. Though different EH units operate based on different mechanisms, a systematic flow can be followed to design an efficient interface circuitry. On the energy supply side of the design (Energy Harvester), electrical characteristics such as the effect of resistive load on power output of the EH unit must be investigated. This is done through loadsweep of the EH under different input stimulus conditions. Additionally, the output power of the electromagnetic PZT EH as a function of the electric current passing through the wire must be determined.

On the demand side (control and wireless units), specifications pertaining to the operational conditions, electrical characteristics, and power consumption of the selected off-the-shelf Microcontroller 
unit must be extracted and analyzed. Ultimately, through a thorough analysis of the information obtained above and reviewing the requirements of the intended application, a compatible circuit interface will be designed for the optimized operation of the sensor node.

\subsection{Current Sensor}

Monitoring the electrical energy consumed by individual appliances in a residential or commercial building requires the deployment of AC current monitoring sensors that will be attached to individual appliances and could non-intrusively measure the current drawn by that given appliance over time. These sensors need to be sensitive, simple and robust, with no external power requirement. Having real-time information regarding the power consumed by individual electronic appliances enables users to monitor and improve the energy consumption efficiency across the building [25].

Stick-on proximity-based PZT current sensors have been reported to be successful candidates for this application. Analogous to the PZT EH, piezoelectric sensors use a permanent magnet placed at the end of a cantilever beam in order to couple with the magnetic field surrounding a current carrying wire. The magnetic field induces a mechanical force on the magnet that would deflect the piezoelectric cantilever producing an alternative electric current [26]. The amplitude of the output signal is proportional to the intensity of the current in the wire and will be sampled by an Analog-to-Digital Converter (ADC). A schematic illustration of a piezoelectric AC current sensor is provided in Figure 2.11 [27].

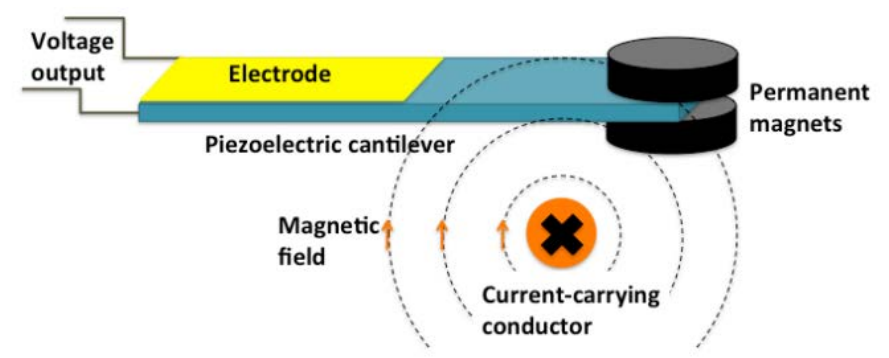

Figure 2.11- Piezoelectric AC current sensor [27]

The sensor chosen for this project was designed at the University of Waterloo's Energy Harvesting Laboratory [4]. The sensor operates at the natural frequency of $781.0 \mathrm{~Hz}$ which is substantially higher than that of the EH. The high resonant frequency ensures a high bandwidth and a linear operation of sensor in 
response to the changes in the intensity of current within the wire. Table 2.2 provides detailed information regarding the design specifications and electrical characteristics of the piezoelectric AC current sensor [4].

Table 2.2- Design specifications of the piezoelectric AC current sensor designed at the UW Energy Harvesting Laboratory

\begin{tabular}{lc}
\hline Specification & Value/Range \\
\hline Sensor operating current range & $10 \mathrm{~A}-200 \mathrm{~A}$ \\
Sensor accuracy within & $1 \% @ 10-100 \mathrm{~A}, 4 \% @ 100-200 \mathrm{~A}$ \\
Operating temperature & $-40{ }^{\circ} \mathrm{C}$ to $80^{\circ} \mathrm{C}$ \\
Output voltage for $10,000 \mathrm{~A}$ & $\pm 1.75 \mathrm{~V}$ \\
Sensor impedance & $55 \mathrm{k} \Omega$ \\
\hline
\end{tabular}

The PZT AC sensor used in this project has been designed for current measurement in single conductors primarily used in power transmission lines (operating current range of 10A to 200A). Although lower currents can also be measured with the above mentioned sensor (through the amplification of the output signal), the sensitivity of the sensor is substantially reduced in this range. The piezoelectric sensor output is a $60 \mathrm{~Hz}$ AC signal that is fed into the input of an Analog-to-Digital Convertor (ADC). As shown in Figure 2.12, sensor's output (converted from AC peak-to-peak to their corresponding RMS values) is linearly proportional to the intensity of current passing through the wire. The signal output from the sensor can be fed directly to the Microcontroller unit for data acquisition, collection and storage. Subsequently, the wireless transmission device can generate data packets that are transmitted through a Radio Frequency (RF) transceiver [28].

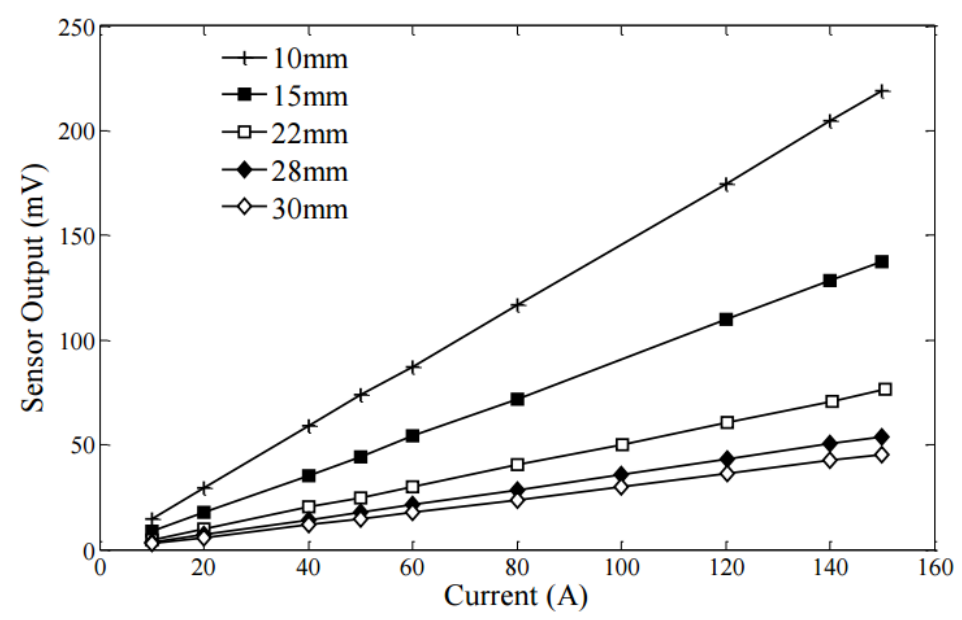

Figure 2.12- Sensor's output (RMS voltage) as a function of the current passing through the wire. Different plots represent different distances between the wire and the sensor [4] 


\subsection{Microcontroller Unit}

Advances in the development of low-cost microcontrollers with enhanced computational power has led to the emergence of low powered wireless sensor nodes designed for novel and innovative applications. In the case of WSN for electricity monitoring applications, the MCU is involved in sensor data acquisition and collection, monitoring the energy state of the node, initializing tasks, and communication with the Radio Frequency transceiver. Implementation of the MCU within a sensor node requires meticulous evaluation of its core hardware as well as its system architecture and design [29].

It is essential to sustain a high level of functionality and performance while simultaneously minimizing the energy consumption at each node. The power consumed by the MCU is minimized through selecting an optimal architecture and also, via implementing a suitable algorithm to control the unit. Other elements such as the selected communication protocol can also play a significant role in the node's overall power consumption.

Nevertheless, with the trade-off present between the energy consumed at the node and the node's performance, an optimized solution can be only achieved by implementing a dynamic energy monitoring and power management system that could minimize the power consumed by the MCU while maintaining its required performance as it undertakes its assigned tasks. Such dynamic functionality provides the system with the ability to make trade-off decisions autonomously depending on the operating conditions.

\subsubsection{Microcontroller Power Consumption and System Life Time}

The microcontroller is one of the most fundamental elements in the operation of wireless sensor nodes and it is responsible for consuming a large portion of the total energy available to a single node. MCU provides a framework based on which operational parameters are adjusted so that the node can function according to the instructions set by the user. The MCU should be able to control the node with the limited power resources available to it in order to extend the system's life time [28].

In the case of nodes operated by batteries, the approximate lifetime of the system is determined based on the total energy stored in the battery and the energy consumed by the MCU for a given process [30]. However, making such assessment in the case of nodes operated by EHs is more complicated. The EH's operation is heavily dependent on its working conditions and therefore, predicting its energy harvesting capabilities over time would be difficult. Thus, achieving an optimized operation is only possible through a dynamic approach using a circuitry as well as an embedded software that are capable of 
monitoring the EH and making decisions regarding the node's operation [31]. Such process, makes the wireless sensor node adaptable to any given environmental and operational condition.

\subsubsection{Data Collection and Processing}

Once data is gathered from the sensor, two different approaches are available to process and transmit the necessary information. Each approach has its own advantages and disadvantages and depending on the application and the amount of data gathered from the sensor, one can be more effective than the other. Depending on the size of the data array obtained from the sensor, data transmission through radio communication may consume greater power compared to the direct on-node data processing. In the first approach, through on-node data processing, the size of data obtained from the sensor is shrunk and therefore, the amount of information required to be transmitted by the radio is reduced, dwindling the power consumed by the node [28].

Contrary to this case, when data acquired by the sensor requires to go through complex processing algorithms that can be only handled by more powerful computers, transmitting all the acquired information obtained from the sensor is recommended [32]. In this case, less energy will be consumed by the MCU onnode data processing and more weight is placed on the wireless transmission of data. Hence, it is established that the performance and power efficiency of the sensor node can be heavily influenced by the existing trade-off between the local on-node data processing and external data processing through transmission of raw data by the radio unit.

\subsubsection{Microcontroller Hardware Parameters}

Selecting the right MCU for a given application is mainly dictated by factors such computational requirements of the sensor node, power consumption, size, cost, and reliability. Through a detailed and comprehensive investigation of application requirements, a Microcontroller tailored to the needs of the sensor network domain and capable of delivering demands of the system is selected. Additionally, through programming the MCU according to the requirements of the intended application, the user can further enhance the efficiency of the MCU by disabling functions available in most off-the-shelf MCUs that are not necessary to the of application under development [33].

Besides having an attractive power profile and computational capabilities, the accessibility of design support tools, and also the availability of software libraries can also significantly impact the selection process. New generations of ultra-low power MCUs can operate with high precision, a low quiescent 
current, and a high operation efficiency. Manufacturers of these MCUs provide the users with access to ample resources and software libraries which would further ease the development of the software platform. Texas Instruments offers a wide range of Microcontroller units paired with sub-GHz Radio Frequency transceivers. The most commonly used MCU solutions for sensor nodes in the research community are the MSP430F247 Microcontroller and CC2430 Microcontroller designed by Texas Instruments. Other solutions such as the ATmega168P and the ATxmega32A4 designed by Atmel have been also reported for the use in self-contained WSN applications.

\subsection{Wireless Transmitter}

Wireless Sensor Networks are comprised of a number of nodes that are provided with a communication getaway where data obtained from the sensor is transmitted to a central hub. Through sending the raw data to the base station for post-processing, the amount of energy consumed by the node through intensive onnode computational processes is reduced. The base station, with a compatible wireless network interface, will collect the data for post processing purposes [34]. Wireless networks are categorized by their topology and network arrangement. The most fundamental topologies are the Mesh structure and the Star structure. Other topologies such as Fully Connected, Ring, Tree, or Bus structures may be used depending on the installation environment, economic considerations, and the intended application of the project [35]. Figure 2.13 [36] depicts the arrangement of the nodes within each fundamental network topology.
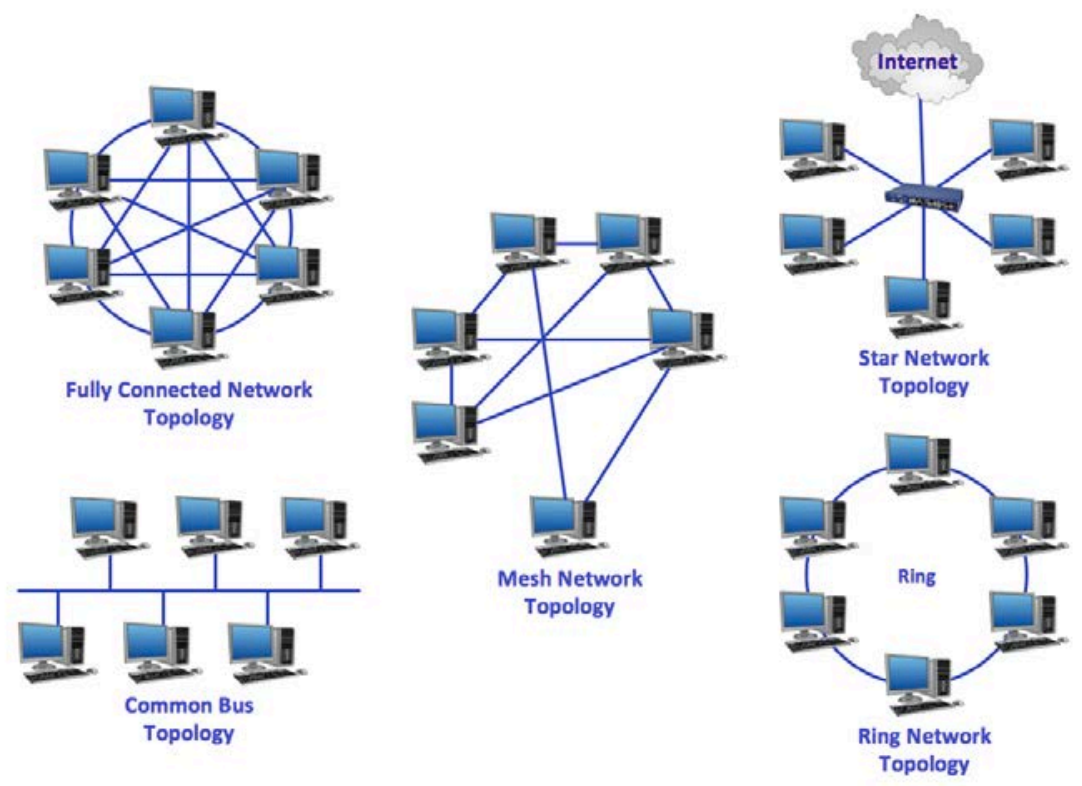

Figure 2.13- Network topologies 
Wireless networks can be also categorized according to their operating range. These categories include the Personal Area Network (PAN) for which a range close to 10 meters is covered by each node, Local Area Network (LAN) covering a range up to a 100 meters, Neighborhood Area Network (NAN) and Wide Area Network (WAN) that are capable of reaching several kilometers [37]. The operational network range of the system is selected according to the intended application of the project. PAN and LAN networks are generally used for applications that are designed to operate within buildings. These networks allow each node to transmit data to the next node or the central hub with minimal power consumption which makes these networks attractive for energy harvesting applications.

Other factors such as network size, number of active nodes, data transmission frequency, and the availability of power source are all important considerations while selecting a suitable network topology and communication technology. In the case of WSN for electricity monitoring within residential and commercial buildings, the Mesh network structure has certain advantages that makes it a top candidate for this application. Within this network structures, nodes can be connected to one another with one or more nodes acting as the hub for Internet getaway. Therefore, the operating range of the complete network can be extended across the building as data packets hop from one node to another [37].

The focus of this project was placed on point-to-point data transmission from one node to another. With the reduced power consumption and the ability to simultaneously connect to up to 20 devices, Bluetooth Low Energy (BLE) is considered as the most suitable candidate for the electricity monitoring application. Each node can be also connected to a computer or a tablet device for data transmission through an Internet getaway. Therefore, given the above advantages, BLE was selected as the wireless network technology in this project

\subsection{System Integration}

Design and development of a wireless sensor node requires the integration of all its building blocks to a single self-contained unit. These components (the EH, electric current sensor, Micro-controller Unit, and the wireless communication device) are integrated within the system according to their specifications so that each part can operate with maximum effectiveness in undertaking its assigned tasks. Interfaces among these modules are developed to enhance the system's flexibility. The integrated system will work as a selfcontained, intelligent, and autonomous unit that can provide real time data regarding the electricity usage by individual appliances within residential and commercial buildings. 


\subsection{Design Requirements}

Upon thorough examination of the current available state-of-the-art systems, and after careful review of some of their inherent limitations, a list of system requirements is proposed that is aimed at improving the current available technology. These requirements are set to tackle some of the critical constraints associated with wireless sensor nodes in order to develop and design a self-contained and non-invasive system for electricity monitoring applications.

1. The unit must be self-contained to eliminate the need for external power sources such as batteries.

2. The unit must be able to operate when mounted on conducting wires carrying a current in the range of 7A to 27A (this range covers the current drawn by most appliances in a residential or commercial building).

3. The unit should be able to capture the peak voltage value of a $60 \mathrm{~Hz}$ sinusoidal signal with an amplitude in the range of $100 \mathrm{mV}$ to $900 \mathrm{mV}$ (the expected sensor output).

Note that we are only concerned about the peak voltage value of the sensor's output signal as it can be correlated to the amplitude of the current passing through the wire.

4. The unit must be able to detect the signal peak within $10 \%$ error range of the nominal value.

5. The unit should be able to wirelessly transmit the processed data to a central communication hub using an accessible network standard.

6. The unit must maintain a read/transmit duty cycle less than 4 minutes (Receiving the data every 4 mins is adequate for the purpose of this application).

\subsection{Summary}

Electromechanical energy scavenging using a PZT EH is considered as the most promising approach in eliminating the need for batteries in sensor nodes. However, the AC output voltage from the EH needs to be rectified, regulated, and conditioned before it can be used by the load circuitry. Using a power conditioning circuitry compatible with both the EH and the control and communication devices, the energy obtained by the EH can be stored and used to power the electronic devices. An optimized solution should be able to monitor the performance of the $\mathrm{EH}$ and use a power management system to minimize the energy consumed by the MCU as its assigned tasks are undertaken. The MCU and wireless device are programmed to acquire, process, and transmit the data from the sensor to the central hub. Once the analog input signal from the sensor is digitized, the processed data can be transmitted using the BLE technology that is considered as the most suitable technology for WSNs within buildings. 


\section{Chapter 3}

\section{Design Approach}

\subsection{Introduction}

The designed wireless sensor node must be able to meet all the set requirements of the electric current monitoring application all the while tackling some of the critical constraints associated with the design of WSNs. Based on the system requirements set in the previous chapter, two design approaches will be introduced here. Each approach entails a number of key advantages and disadvantages. Taking that into consideration, the design most suitable for the electricity monitoring application will be selected and implemented for testing.

We begin this chapter by discussing some of the general considerations for the design of a selfcontained wireless sensor nodes. We will then proceed to discuss the means for meeting the set requirements of the system and will establish the benchmark for testing the circuit. Some of the preliminarily test results are included in this chapter and will be analyzed and investigated in depth in Chapter 4. 


\subsection{System Components’ Technical Validation}

Design and development of an integrated system comprised of different modules requires a careful review of technical specifications of each individual block. Absolute maximum ratings, power generation or consumption characteristics, recommended operating conditions, and other general electrical characteristics pertaining to each module should be studied carefully before instigating the design of the integrated system. Next, these components can be integrated according to their specifications in order for each component to operate with its maximum effectiveness.

We begin this section by providing a summary of each module's behavior and characteristics under various operating conditions. These system components include:

- $\quad$ Piezoelectric Energy Harvester

- $\quad$ Electromagnetic Piezoelectric Sensor

- $\quad$ Microcontroller Unit

- Wireless Transceiver

Each component is characterised independently and autonomously. The results obtained here are used in the development of two system designs that are presented subsequently.

\subsubsection{Piezoelectric Energy Harvester}

Electromechanical EHs are generally tested and characterized using an electrodynamic shaker under a base excitation that mimics the desired operating condition. The shaker can be set to operate at a given excitation frequency and base acceleration. By constructing conditions similar to that of EH's operating environment, the accurate operation of the fabricated EH can be validated. Additionally, the technical characteristics of the unit including its RMS power output under different excitation intensities, its output impedance, and other performance measures of the unit are obtained throughout this process.

The EH's output voltage Frequency Response Function (FRF) is measured through a frequency sweep from $40 \mathrm{~Hz}$ to $80 \mathrm{~Hz}$ at a constant base acceleration. Experimental results indicate a natural frequency of $60.6 \mathrm{~Hz}$ associated with this unit. The resonance frequency of $60.6 \mathrm{~Hz}$ taken from the EH's FRF plot (provided in Figure 3.1(b)) validates the similarity of the natural frequency of the EH and that of the North American $60 \mathrm{~Hz}$ power line frequency. The matched frequency between the $\mathrm{EH}$ and the electric current of the North American power lines leads to the optimal operation of the EH. 


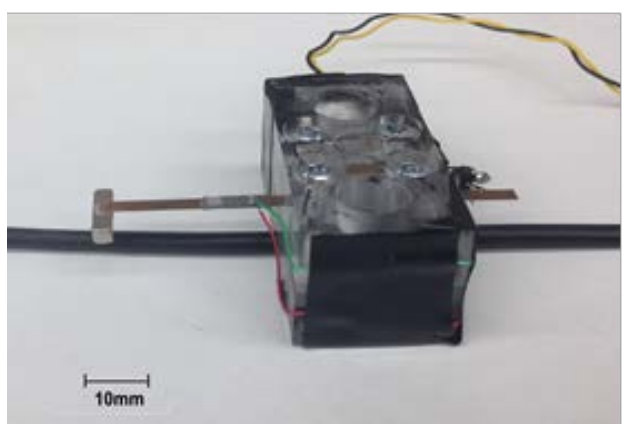

(a)

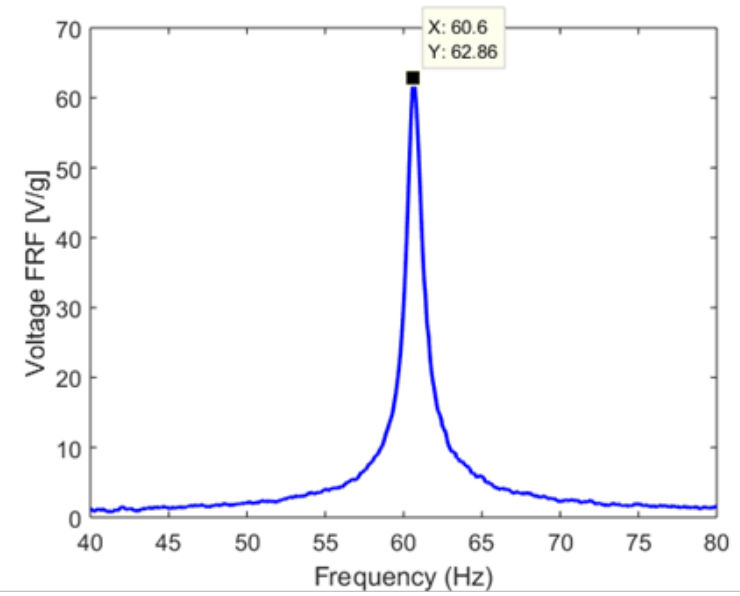

(b)

Figure 3.1- (a) A photograph of the electromechanical piezo-electric energy-harvesting unit. (b) Frequency Response Function (FRF) of the PZT EH with peak voltage at the resonance frequency of $60.2 \mathrm{~Hz}$

Although a number of analytical models exist that can predict the output impedance of the EH, the inadequate accuracy of these models makes it difficult to obtain a precise and reliable result. EH is fabricated manually and a number of factors associated with the fabrication process (such as the additional stiffness caused by the adhesives used) cannot be accounted for by analytical methods. Therefore, the optimal resistive load, $\mathrm{R}_{\mathrm{opt}}$, is measured using an experimental approach.

Given that the EH used throughout this project is designed for an exclusive use in electricity monitoring applications, efforts are directed towards obtaining its performance parameters under its expected operating condition. Therefore, by placing the EH on a conducting wire connecting an electric load (a home appliance) to an electric outlet, the end use working condition of the unit is replicated. The $\mathrm{EH}$ is mounted at a distance of $6 \mathrm{~mm}$ above the wire that is set to conduct a $60 \mathrm{~Hz}$ AC electric current within a range of $7 \mathrm{~A}$ to $27 \mathrm{~A}$.

Thru connecting a potentiometer across the EH and by monitoring the voltage drop across the load (as shown in Figure 3.2), the power output of the EH as a function of the load is measured and plotted (Figure 3.3). The load resistance for which the output power from the EH peaks marks the $\mathrm{R}_{\mathrm{opt}}$. The optimal power transfer resistance indicates the output impedance of the $\mathrm{EH}$ as proven in the following circuit analysis of the system [38]: 


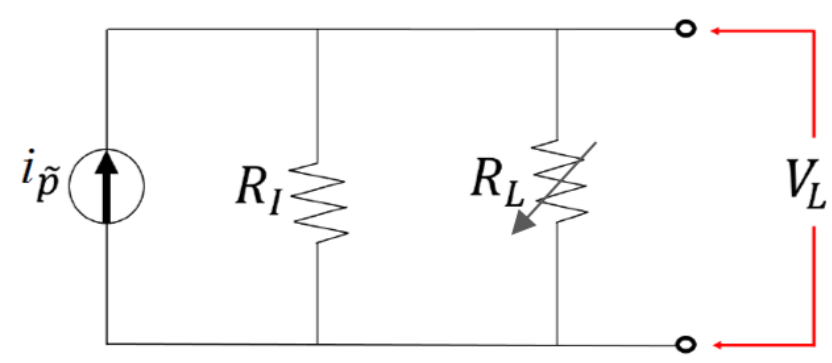

Figure 3.2- EH’s output power analysis circuit configuration

$$
\begin{aligned}
& i_{L}=\frac{i_{P}}{\left(\frac{R_{I} \times R_{L}}{R_{I}+R_{L}}\right)} \\
& P_{L}=R_{L} \times i_{L}^{2}=\frac{i_{P}^{2}}{\left(\frac{R_{I}^{2} \times R_{L}}{\left(R_{I}+R_{L}\right)^{2}}\right)}
\end{aligned}
$$

To obtain the load for which power output is maximized, the derivative of $P_{L}$ is set to zero. Therefore,

$$
\begin{gathered}
\frac{d}{d R_{L}}\left(P_{L}\right)=0 \\
\frac{d}{d R_{L}}\left(\frac{\left(R_{I}+R_{L}\right)^{2}}{R_{I}^{2} \times R_{L}}\right)=0 \\
R_{I}=R_{L}
\end{gathered}
$$

The above analysis indicates that the output power obtained from the $\mathrm{EH}$ is maximized at the point where the resistive load across the unit $\left(\mathrm{R}_{\mathrm{L}}\right)$ equalizes the output resistance of the $E H\left(\mathrm{R}_{\mathrm{I}}\right)$. Therefore, the output resistance of the EH is determined using the load-sweep method for which the power output of the unit is measured and graphed as different resistive loads are connected across the EH . As shown in Figure 3.3, the resistive load for which the output power of the EH peaks corresponds to the output resistance of the $\mathrm{EH}(1.4 \mathrm{M} \Omega)$. It is important to note that this value is independent of changes in the amount of electric current passing through the conducting wire. 


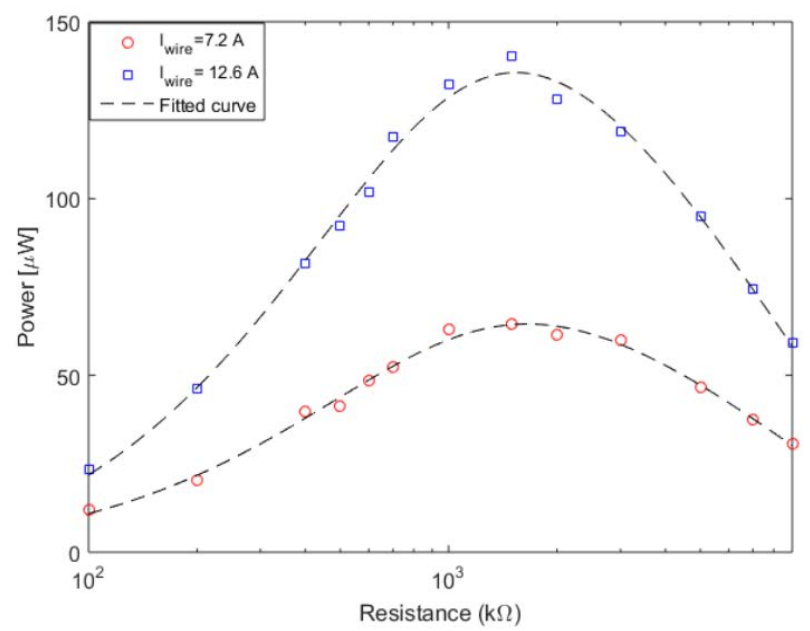

Figure 3.3- Output power of the EH unit measured by the load-sweep of the EH mounted on a wire carrying different electric current values

Another important consideration before moving ahead with the design of an interface circuitry is the analysis of the EH's power output as a function of the electric current passing through the conducting wire. As per the application requirements set for the system, it was established that the current will be in the range of $7 \mathrm{~A}$ to $27 \mathrm{~A}$. In order to determine the power output from the $\mathrm{EH}$ within this operating range, the EH's power output as a function of the current passing through the wire is measured and plotted in Figure 3.4. The obtained measurements indicate that for currents ranging from 7A to 27A, the maximum RMS power generated by the EH unit will be in the range of $50 \mu \mathrm{W}$ to $400 \mu \mathrm{W}$.

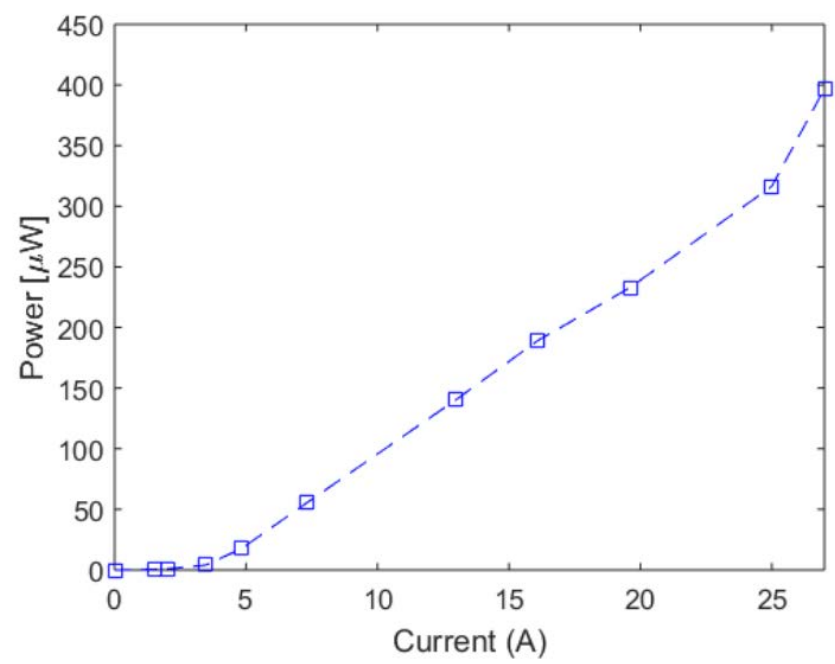

Figure 3.4- EH's RMS power output measurement at its optimum load and as a function of the current passing through the wire 


\subsubsection{Piezoelectric Current Sensor}

The Piezoelectric electric current sensor provided to us by the University of Waterloo's Energy Harvesting team [4] is primarily designed for monitoring the electric current passing through the power transmission lines. Therefore, for the case of single conductors carrying a current in the range of 7A to 27A, the signal output from the sensor device would be weak, noisy, and more difficult to detect. Thus, considering the specified operating range for the wireless sensor node, a new sensor must be designed and developed that could operate according to the requirements of the system. In order to maintain the progress of this work whilst the new sensor is being designed, the anticipated signal output from this sensor is replicated using a function generator.

\subsubsection{Microcontroller and Wireless Transmitter}

System requirements provided in section 2.8 were used as the benchmark for the selection of a suitable single-chip Microcontroller with an onboard RF transceiver. The search for the solution was narrowed down based on the power consumption profile of the available off-the-shelf Wireless MCUs. Furthermore, the ease of integration with the EH was used to finalize the decision leading to the selection of two Wireless MCU solutions (ATMEGA1281R212 by Atmel and CC2640R2F by Texas Instruments) as the final candidates. Both solutions operate under a very low Active Mode current, and have been designed to target WSN applications. They are both compatible with external sensors, can digitize the collected analog data autonomously, and transmit data packets over a wireless network. The following chart provides specific details of the technical specifications for both solutions.

Table 3.1- Feature Analysis ATMEGA1281R212 vs. CC2640R2F [39] [40]

\begin{tabular}{|c|c|c|}
\hline $\begin{array}{l}\text { Vendor Name \& } \\
\text { Part Number }\end{array}$ & $\begin{array}{c}\text { Atmel } \\
\text { ATMEGA1281R212 }\end{array}$ & $\begin{array}{l}\text { Texas Instruments } \\
\text { CC2640R2F }\end{array}$ \\
\hline Description & Wireless MCU & Wireless MCU \\
\hline Processor Technology & ARM ${ }^{\circledR}$ Cortex ${ }^{\circledR}-\mathrm{M} 0$ & ARM ${ }^{\circledR}$ Cortex ${ }^{\circledR-M 3}$ \\
\hline Flash (KB) & 128 & 128 \\
\hline RAM (KB) & 8 & 20 \\
\hline Clock Speed (MHz) & 16 & 48 \\
\hline Active Mode Current $(\boldsymbol{\mu} \mathbf{A} / \mathbf{M H z})$ & $500 @ 1.8 \mathrm{~V}$ & 61 \\
\hline Standby Current $(\boldsymbol{\mu} \mathrm{A})$ & 0.1 & 1.1 \\
\hline ADC Resolution & 10-Bit & 12-Bit \\
\hline ADC Sampling Frequency(ksamples/s) & - & 200 \\
\hline Wireless Technology & 6LoWPAN, Zigbee ${ }^{\circledR}$ & Bluetooth low energy(BLE) \\
\hline Throughput (Max) (Mbps) & 1 & 5 \\
\hline Active-Mode TX Current (+5 dBm) (mA) & 17 & 9.1 \\
\hline
\end{tabular}


In the view of design considerations and the intended end use application of the self-contained wireless sensor node, and after conducting a thorough requirement/specification analysis of all available technologies (including both the ATMEGA1281R212 designed by Atmel technologies and CC2640R2F designed by Texas Instruments) CC2640R2F is selected as the most suitable solution for the purpose of this project. Power consumption profile, the availability of development tools and libraries, ease of integration with the $\mathrm{EH}$, and the available on-board wireless technology are some of the considerations that were factored in during this selection process. The ultra-low power consumption of the CC2640R2F in Active Mode and also, its reduced current consumption in Standby Mode makes it an excellent candidate for energy harvesting applications. The device contains a 32-bit $\mathrm{ARM}^{\circledR}$ Cortex $^{\circledR}-\mathrm{M} 3$ core that runs at $48 \mathrm{MHz}$. The unit's Bluetooth Low Energy BLE controller and its host libraries are embedded in the Read Only Memory (ROM) which improves the overall power consumption of the unit. It also frees up a significant amount of flash memory for any required on board data processing [39].

Development tools provided by Texas Instruments including the Code Composer Studio (an Integrated Development Environment (IDE) exclusive to Texas Instruments products) is particularly helpful in configuring the Wireless MCU according to the design requirements. This development environment includes additional software frameworks and reference design libraries that provide a starting point for application development on the device. Figures 3.5 and 3.6 show the functional block diagram and the package configuration of the CC2640R2F Wireless MCU.

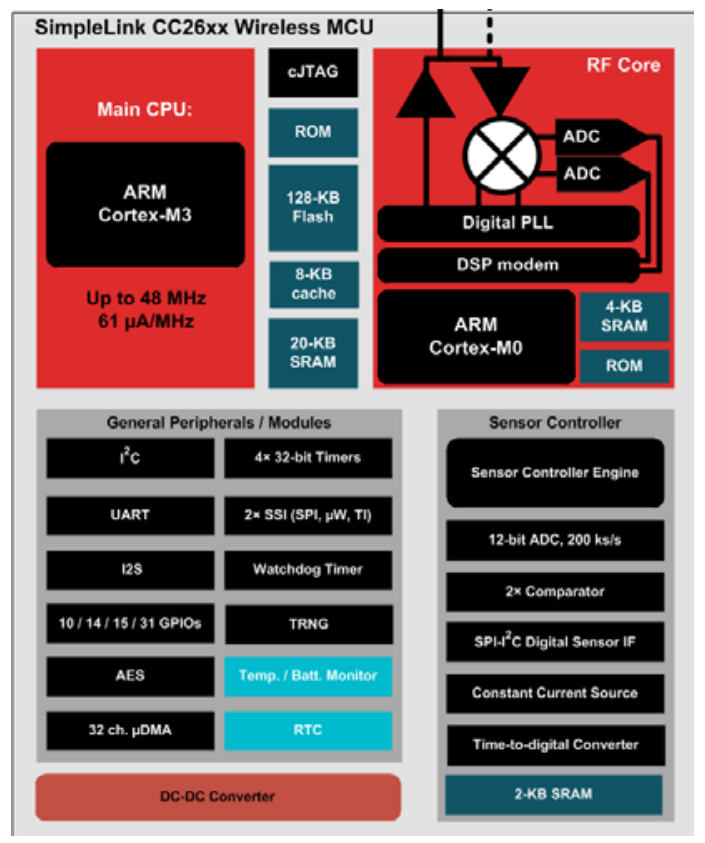

Figure 3.5- Functional block diagram of the CC2640R2F Wireless MCU 


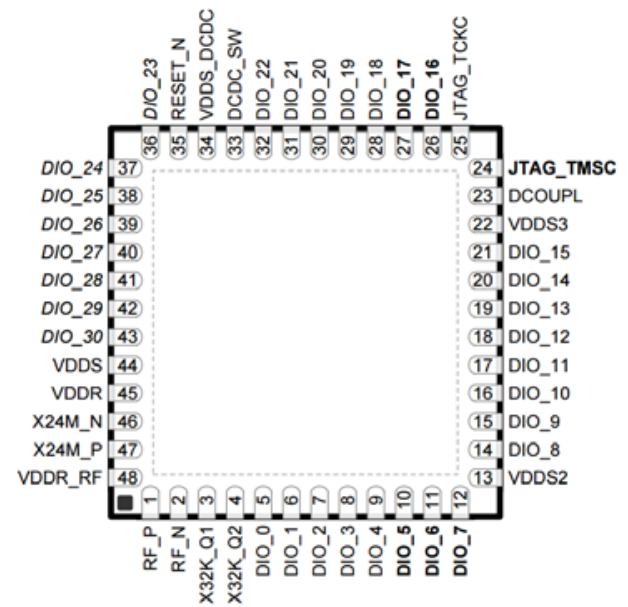

Figure 3.6- 48-pin CC2640R2F Wireless MCU package $(7.00 \mathrm{~mm} \times 7.00 \mathrm{~mm}$ )

The following chart includes the recommended operating conditions and the absolute maximum ratings of the CC2640R2F unit. Failure to operate within these recommended ranges imposes additional stress on the device and may lead to permanent damage. Therefore, these values are taken into careful consideration throughout the design of the power conditioning circuit.

Table 3.2- Operational condition of the CC2640R2F Wireless MCU [39]

\begin{tabular}{|c|c|cc|c|}
\hline \multicolumn{2}{|c|}{} & Min & Max & Unit \\
\hline $\begin{array}{c}\text { Recommended } \\
\text { Operating Conditions }\end{array}$ & supply voltage (VDDS) & 1.8 & 3.8 & V \\
\hline $\begin{array}{c}\text { Absolute Maximum } \\
\text { Ratings }\end{array}$ & supply voltage (VDDS) & -0.3 & 4.1 & $\mathrm{~V}$ \\
\cline { 2 - 5 } & Voltage on ADC input (Vin) & -0.3 & VDDS & V \\
\hline
\end{tabular}

\subsubsection{Summary of Technical Specifications}

Through a careful review of technical specifications linked to each building block of the system, the electrical characteristics needed to develop interfaces among these modules are obtained. Using the acquired information, different blocks are integrated with one another according to their specifications in order for each component to operate with its maximum effectiveness. 


\subsection{Design Methodology}

Following the design requirements, and after a thorough review of technical specifications associated with each building block of the system obtained in the previous section, two design methodologies are proposed here. Both designs are capable of meeting the attributed specifications of the $\mathrm{EH}$, the sensor, and the Wireless MCU. In the following sections, the two designs are compared based on their merits, advantages, and disadvantages. At last, the most suitable design approach is selected for implementation and further testing.

Design I focuses on transforming the unstable energy harvested from the EH into a regulated and stable source of energy capable of powering the control and radio circuitry. Once the Wireless MCU is powered using this stable output from the power conditioning circuit, it will execute its given instructions to accomplish the system's objectives.

Design II takes advantage of the power management methods to develop an intelligent and dynamic system capable of monitoring the operation of the EH in order to enhance the MCU's power consumption efficiency. The system will obtain, process, and transmit the sensory information once adequate amount of energy for undertaking these tasks are available within the storage unit. Such dynamic functionality allows the system to be more flexible in its operation as changes take place in the surrounding environment of the sensor node.

In each case, we begin by providing a detailed account of each design's blueprint. We will then move forward to introduce the complete circuit design as well as the embedded software developed to run the system. Based on the preliminary test results and analysis of the complete system in each case, key advantages and disadvantages of each approach are identified. Using the information, the design most suitable in meeting the system's requirements is selected and implemented for testing.

\subsubsection{Design Approach I}

The output voltage of the $\mathrm{EH}$ is vulnerable to the changes in the ambient environment and thus unstable as a direct power source to the sensor node. Therefore, a highly efficient interface circuitry is required between the EH and the rest of the system. The design strategy proposed here, focuses on the development and optimization of an interface circuitry capable of transforming the unsteady output voltage from the EH into a regulated and stable source of energy. This stable source is used to power the Wireless MCU device in order for it to undertake its assigned tasks. 
To achieve the above objectives, the voltage output from the EH is rectified using a Voltage Rectifier. To prevent the cold start of the circuit and to avert any current leakages from the storage unit during the initial segment of the charging process, the MCU and the voltage regulator are disconnected from the rest of the circuitry until a certain voltage across the storage unit is reached (1.7V). At this point the MCU and voltage regulator will be reconnected to the power source and the system's operation will begin. Failure to disconnect these components during the initial charging process leads to a cold start of the circuit and the collapse of the sensor node unit. Figure 3.7 shows the system block diagram for the design approach I.

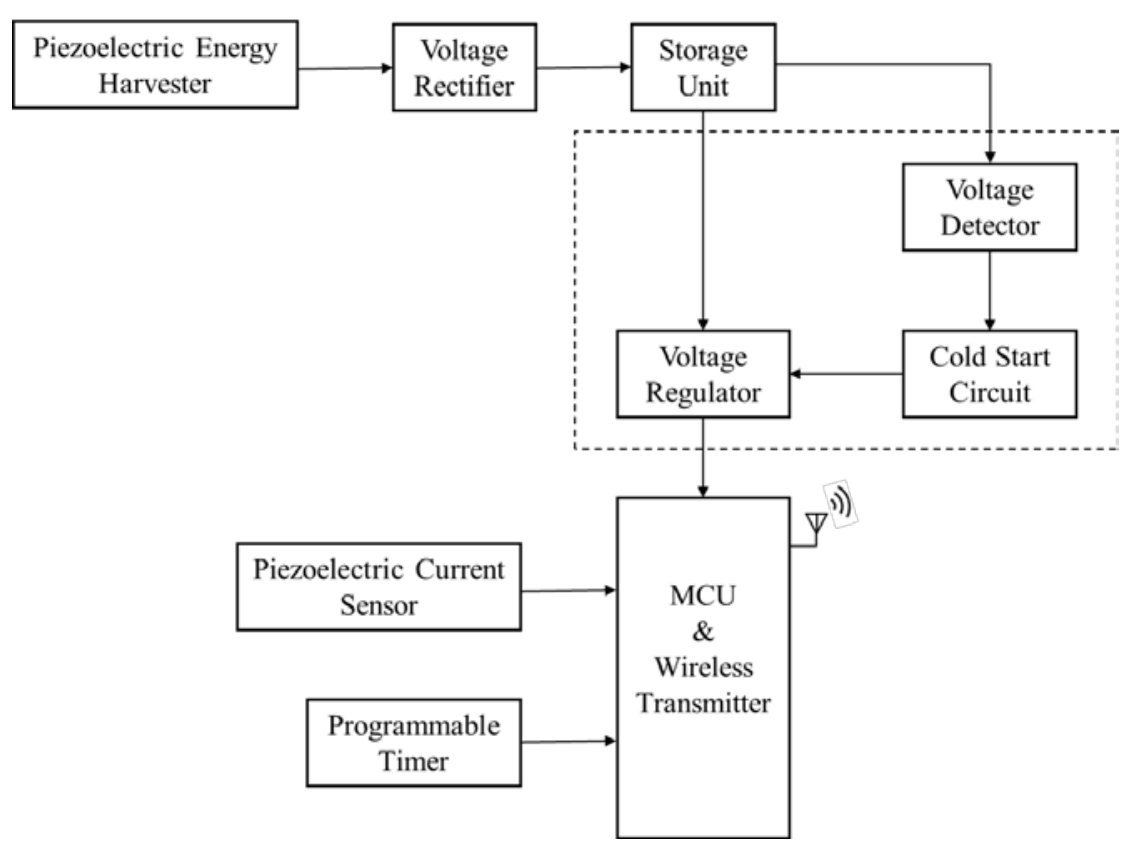

Figure 3.7- Design I architecture

\subsubsection{Circuit Design}

As indicated in section 3.2.1, for currents ranging from 7A to 27A, the maximum RMS power generated by the $\mathrm{EH}$ unit will be in the range of $50 \mu \mathrm{W}$ to $400 \mu \mathrm{W}$. However, the maximum energy from the EH can only be drawn when the impedance of the interface circuitry equals the output impedance of the EH (550Kohms). Nevertheless, due to its negligible impact, and also following the defined and narrow scope of this project, the impedance matching between the two units is postponed for future works.

Figure 3.8 shows the circuit schematic for Design I. Due to the current leakage of the TPS61097A voltage regulator under low input voltages, without the implementation of a cold start circuit, the voltage 
across the $100 \mu \mathrm{F}$ storage capacitor cannot go beyond $0.7 \mathrm{~V}$. To combat this problem, a voltage divider coupled with a multistage amplifier is used to disconnect the voltage regulator from the ground node until a minimum voltage of $1.7 \mathrm{~V}$ is obtained across the $100 \mu \mathrm{F}$ capacitor. During this period, the TPS61097A voltage regulator will function under the bypass mode providing a direct connection path between the bridge rectifier and the load capacitor $(1000 \mu \mathrm{F})$. Once the $100 \mu \mathrm{F}$ capacitor reaches $1.7 \mathrm{~V}$, the multistage amplifier will turn on the M3 transistor (N-Channel MOSFET BS170) to create a path between the voltage regulator's ground node and the common ground of the circuit. At this point, the voltage regulator will be turned ON and will provide an output voltage of $3.3 \mathrm{~V}$ that will be fed to the $1000 \mu \mathrm{F}$ load capacitor.

The TPL5100 programmable timer and the Wireless MCU will be turned ON once the load capacitor is full. The MCU will be kept in the Shutdown Mode where its CPU, SRAM, Wireless Transmitter and Clock are all turned off. In this mode, the unit will consume a minimal current of 150nA and can be only woken up through a wake up signal from an outside source. This signal is provided by the TPL5100 programmable timer which will activate the device for subsequent ADC measurement and data transmission.

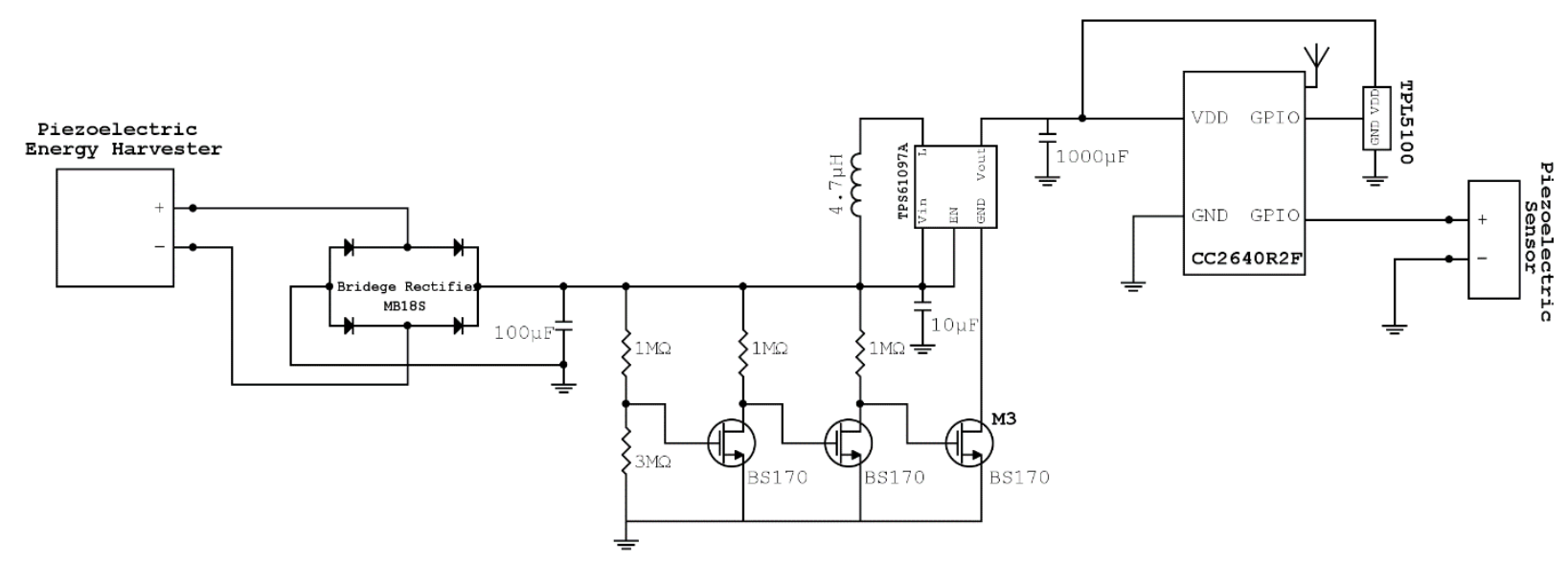

Figure 3.8- Design I circuit schematic

The duty cycle and operating frequency of the system is adjusted by the external programmable timer. TPL5100 will replace the MCU’s internal timer which will allow the MCU to remain in the complete Shutdown Mode during idle periods leading to a substantial reduction in the energy consumed by the MCU. Through analyzing the initial charging and also the recharging behavior of the load capacitor under different charging conditions, an operating duty cycle is selected that would soundly work under all possible circumstances. Figure 3.9 shows the charging behavior of the $1000 \mu \mathrm{F}$ capacitor while the EH was placed on wires conducting a current of 7.2A and 12.6A. 


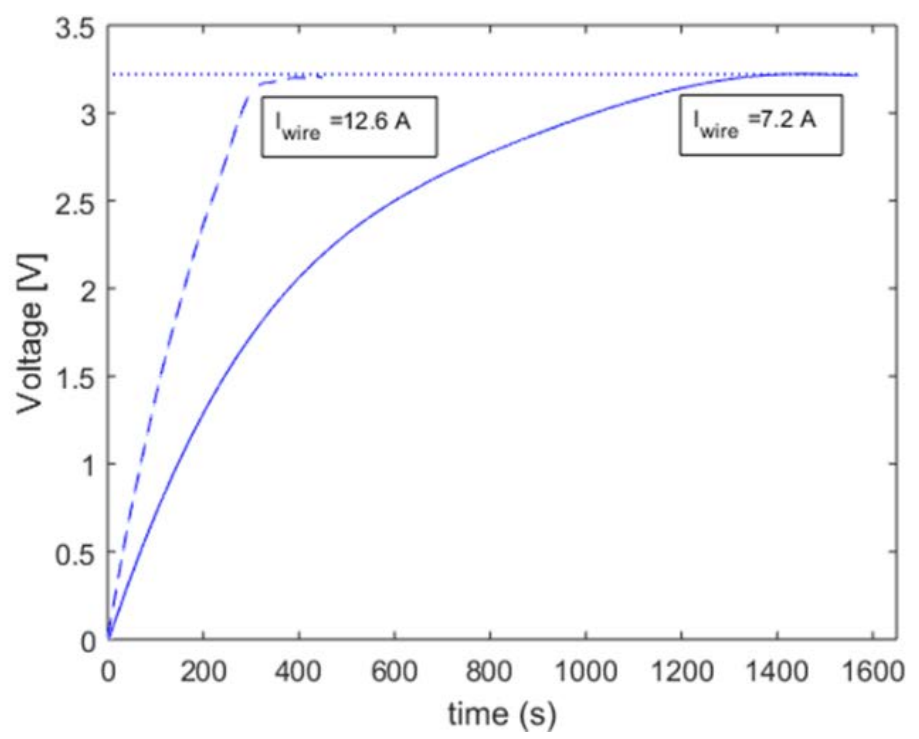

Figure 3.9- The charging behavior of the $1000 \mu \mathrm{F}$ capacitor under different input conditions.

\subsubsection{Embedded Software Development}

The Microcontroller unit must be programmed according to the application requirements in order for the wireless sensor node to undertake function properly. The embedded software for the electricity monitoring application is developed and loaded onto the MCU using the Code Composer Studio (CCS), an Integrated Development Environment (IDE) provided by Texas Instruments.

Figure 3.10 shows the application flow chart diagram developed for Design I. The chart indicates the algorithms and processes used to control the MCU's operation. The process begins by a wake up signal from the TPL5100 unit. This unit is connected to a General Purpose Input / Output (GPIO) pin that has been set to trigger the MCU. This trigger can activate the device on the positive edge of a signal. The script for this defined function is as follows:

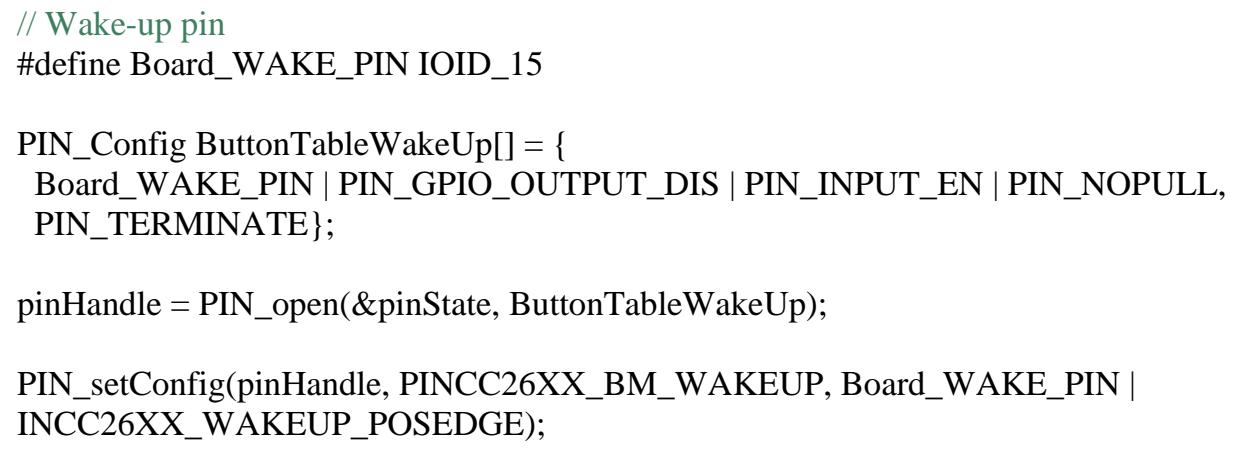




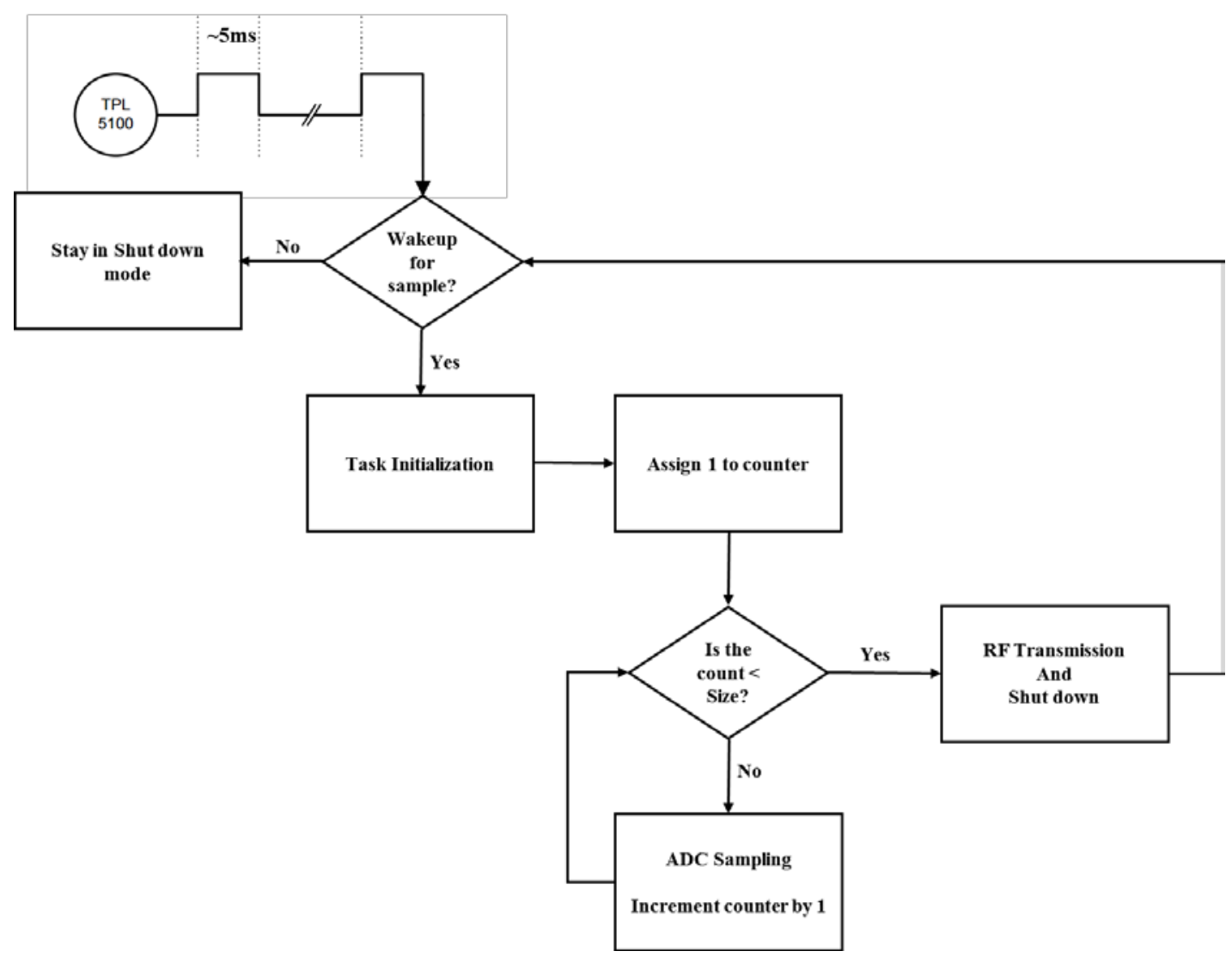

Figure 3.10-Wireless MCU process flow chart

Once the device is woken up from the Shutdown mode, the MCU's operation will continue by setting the ADC and radio parameters. The ADC is set to obtain 15 samples from the sensor output. These samples are evenly spaced out to cover one complete period of the $60 \mathrm{~Hz}$ signal from the sensor device. To achieve this task, the delay time between successive sampling is calculated and set to be $111.1 \mu \mathrm{s}$. The script used for ADC sampling is as follows:

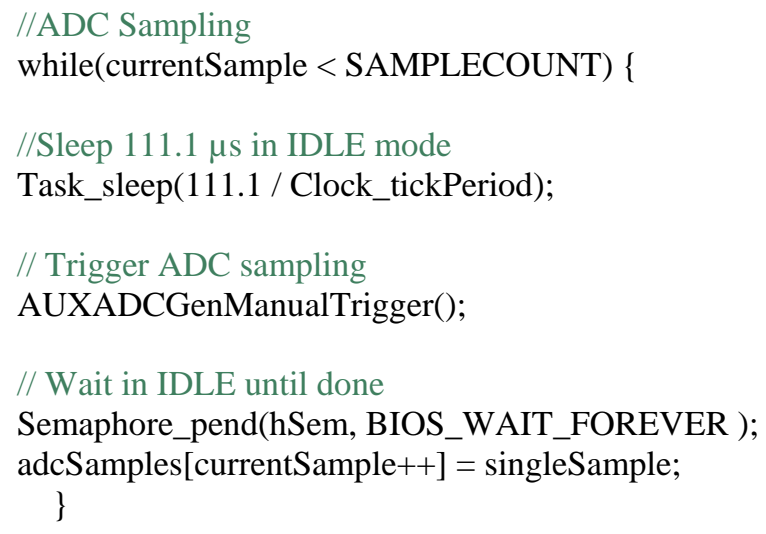


Through obtaining data points from one complete period of the sensor's output, the signal can be reconstructed at the receiver hub where further computational analysis can provide information regarding the signal's amplitude, phase change and other attributes. Figure 3.11 resembles the sampling procedure undertaken by the ADC module. The sampling clock triggers an ADC measurement every 111.1 $\mu$ s.
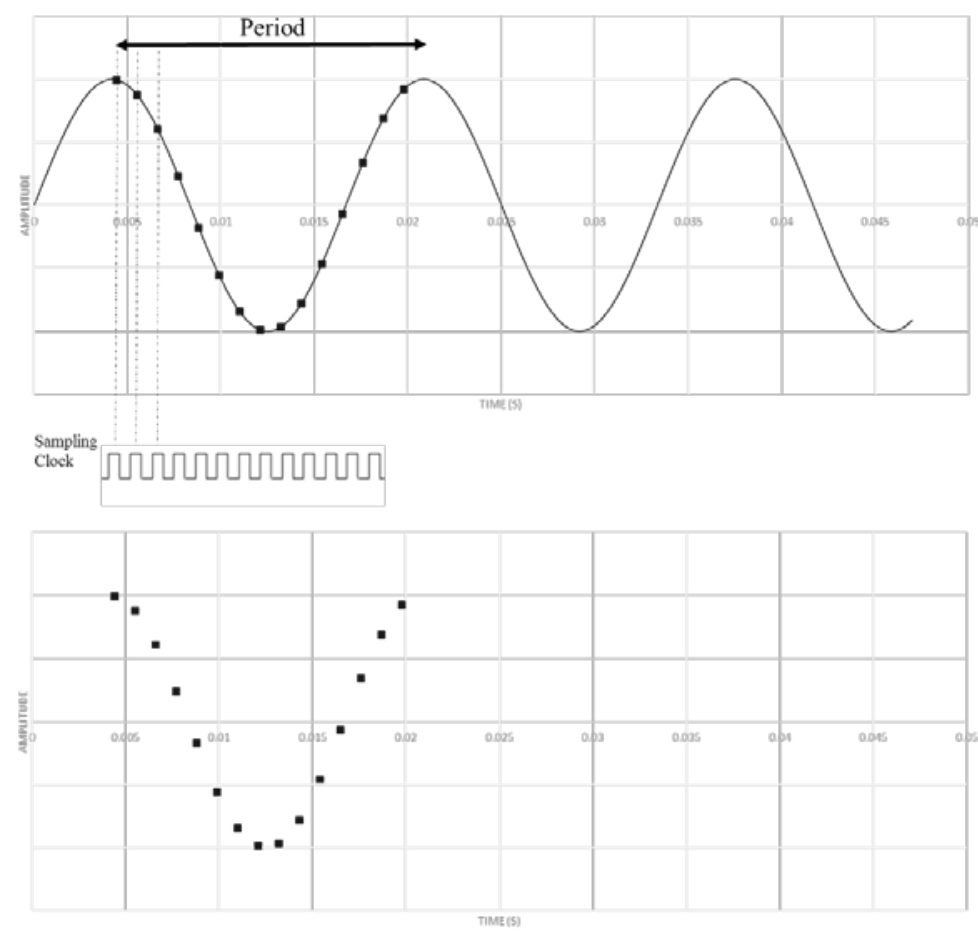

Figure 3.11- ADC sampling procedure

CC2640R2F has a 12-Bit ADC resolution and the data obtained from its measurements are stored in a 16-Bit array. The RF core can assemble the information bits to a packet structure with an 8-Bit encoder. In order to transmit the data, a bit conversion process must be undertaken where the ADC measurements stored in a 16-Bit array are broken down to two 8-Bit arrays. In order to prevent any information loss, the high byte and low byte are stored in two 8-Bit arrays and transmitted individually. The two transmitted arrays are used to reconstruct the original value at the receiver hub. The script used for converting the 16Bit array into two 8-Bit arrays is as follows:

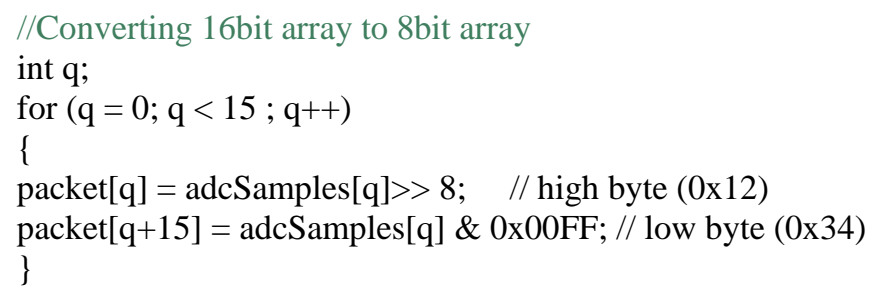


After 8-Bit arrays are constructed, the RF core will transmit the assembled data packets. Once the Wireless MCU is paired with the receiver device (the receiver device needs to be verified through a PIN matching process), these data packets are transmitted at the frequency of $2.4 \mathrm{GHz}$. Next, the ADC and RF modules will be disabled and the device will go to a complete Shutdown mode for maximum power conservation. It is important to note that a number of power modes are available by the MCU that allow the application developer to adjust the power consumption of the unit according to the application needs. Table 3.3 provides a brief overview of these power modes including the state and the performance of some of the MCU's components in each mode.

Table 3.3- CC2640R2F’s power modes and power management features [39]

\begin{tabular}{|l|c|c|c|c|}
\hline \multirow{2}{*}{ Mode } & \multicolumn{3}{|c|}{ Software Configurable Power Modes } \\
\cline { 2 - 5 } & Active & Idle & Standby & Shutdown \\
\hline CPU & Active & Off & Off & Off \\
\hline SRAM & On & On & Duty Cycled & Off \\
\hline Radio & On & On & Available & Off \\
\hline Wake up on RTC & Available & Available & Available & Available \\
\hline Wake up on Pin Edge & Available & Available & $1 \mu \mathrm{A}$ & $0.15 \mu \mathrm{A}$ \\
\hline Current & $1.45 \mathrm{~mA}+$ & $550 \mu \mathrm{A}$ & & \\
\hline
\end{tabular}

In Standby mode, all active peripherals are clocked. Therefore, an interrupt event can be set using the Real-Time Clock (RTC) to bring the processor back to the active mode. Nonetheless, the power dissipated while the system is in the Standby mode is substantially high compared to the power output obtained from the EH unit and thus, this mode is not suitable for energy harvesting applications.

In order to further reduce the power dissipation during inactive segments of the operation, the device can be placed in a complete Shutdown mode where the CPU, SRAM, Radio and most importantly, the CPU's clock are all inactive. In such case, the device can be only woken up using an external interrupt. The extremely low power consumption of the unit under this mode makes this configuration most desirable for energy harvesting applications where effective energy management is of utmost importance.

As it is indicated in the Table 3.3, in Shutdown mode, the device is turned off entirely. Any Input/Output pin will be latched with its value as the system enters the Shutdown mode. A selected GPIO pin is set to function as a reset trigger to wake up the device by a signal interrupt. Such interrupts can be 
generated using an external clock, a voltage comparator, or a voltage detector depending on the application requirement of the system.

\subsubsection{Design Approach II:}

Changes in the performance of the $\mathrm{EH}$ as a result of variations in operating conditions can lead to the instability and flux in the amount of energy produced by the EH. It is therefore difficult to predict the power output of the EH once the sensor node is mounted in place for the end use. Power outages are also inevitable that can completely disrupt the operation of the EH. Such disruptions can result in a system shutdown where no information can be transmitted from the sensor node. Therefore, to address these issues, a dynamic power management system capable of active monitoring of the EH unit is implemented.

The CC2640R2F device supports a number of power modes that enables users to minimize the power consumed by the Wireless MCU by adjusting the CPU's operating mode (Table 3.3). Through implementation of a dynamic power management feature where the operating mode of the Wireless MCU is adjusted according to the performance of the $\mathrm{EH}$, the efficiency and effectiveness of the sensor node can be considerably improved. Additionally, by replacing the voltage regulator with the internal on-chip DCDC converter available on CC2640R2F devices, the power consumption efficiency of the unit can be further enhanced. The on-chip DC-DC converter will power the MCU as long as its input voltage is within the operational range of the device (indicated in Table 4).

Figure 3.12 shows the Design II system architecture. The voltage detector in this case directly regulates the MCU's operation by triggering the ADC which in turn adjusts the operating duty cycle of the Wireless Sensor Node according to the performance of the EH.

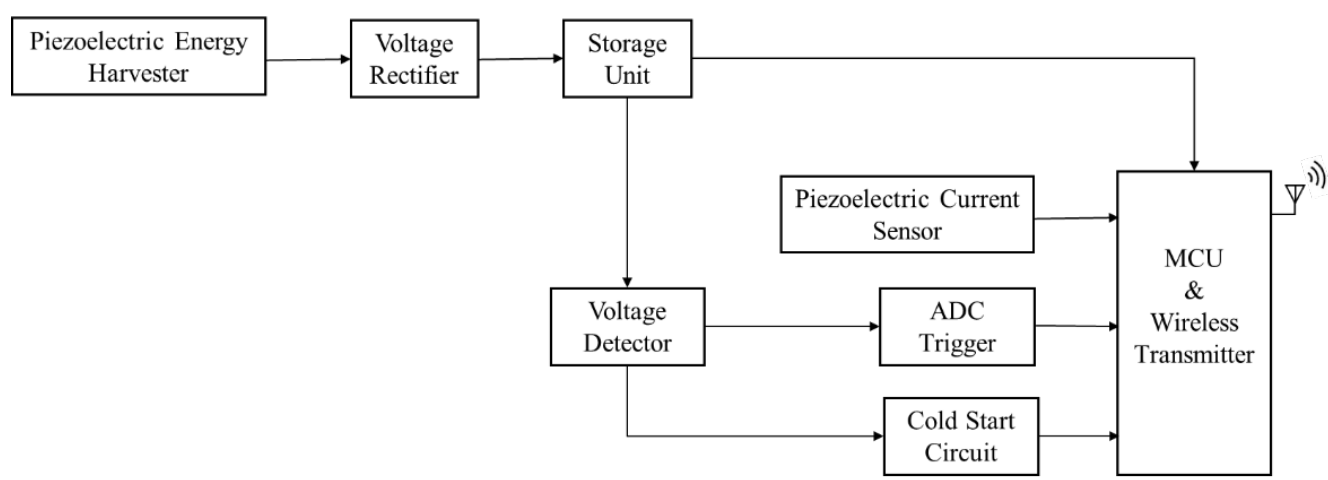

Figure 3.12- Design II architecture 


\subsubsection{Circuit Design}

Implementing a dynamic power management feature requires constant monitoring of the system's power source $(\mathrm{EH})$. In this case, the selected storage unit has been chosen to be large enough to store enough energy for only a single data measurement and transmission activity. The $330 \mu \mathrm{F}$ storage capacitor is directly connected to the Wireless MCU. As demonstrated in Figure 3.13, by replacing the voltage regulator with the internal on-chip DC-DC converter and adjusting the required pin connections, any input voltage within the range of $1.8 \mathrm{~V}$ to $3.8 \mathrm{~V}$ connected to the VDDS pin can effectively power the device.

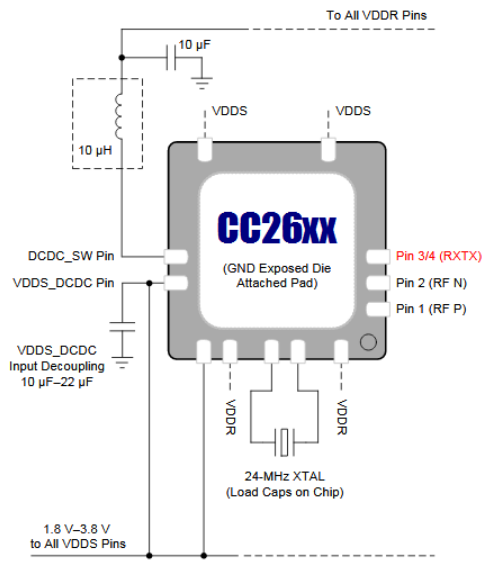

Figure 3.13-Supply voltage configuration for internal DC-DC regulator

During the charging process of the storage capacitor, Wireless MCU is disconnected from the ground node in order to prevent any unintended current leakages. This will allow the $330 \mu \mathrm{F}$ capacitor to reach 3V whilst the CC2640RF device remains disabled. Meanwhile, the voltage divider's output is delivered directly to the gate of the M1 transistor (Figure 3.14) with the addition of a small $10 \mu \mathrm{F}$ capacitor to stabilize the node. As the voltage across the storage unit approaches $3 \mathrm{~V}$, the output provided by the voltage divider will turn on the M1 transistor. Consequently, the multistage amplifier will abruptly reconnect the CC2640RF to the ground node leading to its activation. The MCU in turn will turn on and will follow its instructions to perform an ADC measurement and to transmit the acquired data to the central hub.

The energy required for the system to undertake this process is directly retrieved from the $330 \mu \mathrm{F}$ storage capacitor. As a result, the voltage across the capacitor will drop abruptly to below $2 \mathrm{~V}$ upon completion of a single ADC read and transmit process. At this instant, the MCU will enter the Shutdown mode. The process described above will be repeated in the same order until a new trigger signal activates the read and transmit process. Accordingly, the new design completely eliminates the need for a clock within the system to adjust the operating duty cycle. The duty cycle of this dynamic design is therefore 
adjusted through monitoring the status of the EH. During periods at which EH is scavenging more energy from the conducting wire, the duty cycle of operation is increased leading to a more frequent signal transmission. On the contrary, when the current passing through the wire is low and the EH is unable to produce as much energy to feed the sensor node, the frequency of operation is decreased (reduced duty cycles). Figure 3.14 illustrates the circuit schematic of this new design in details.

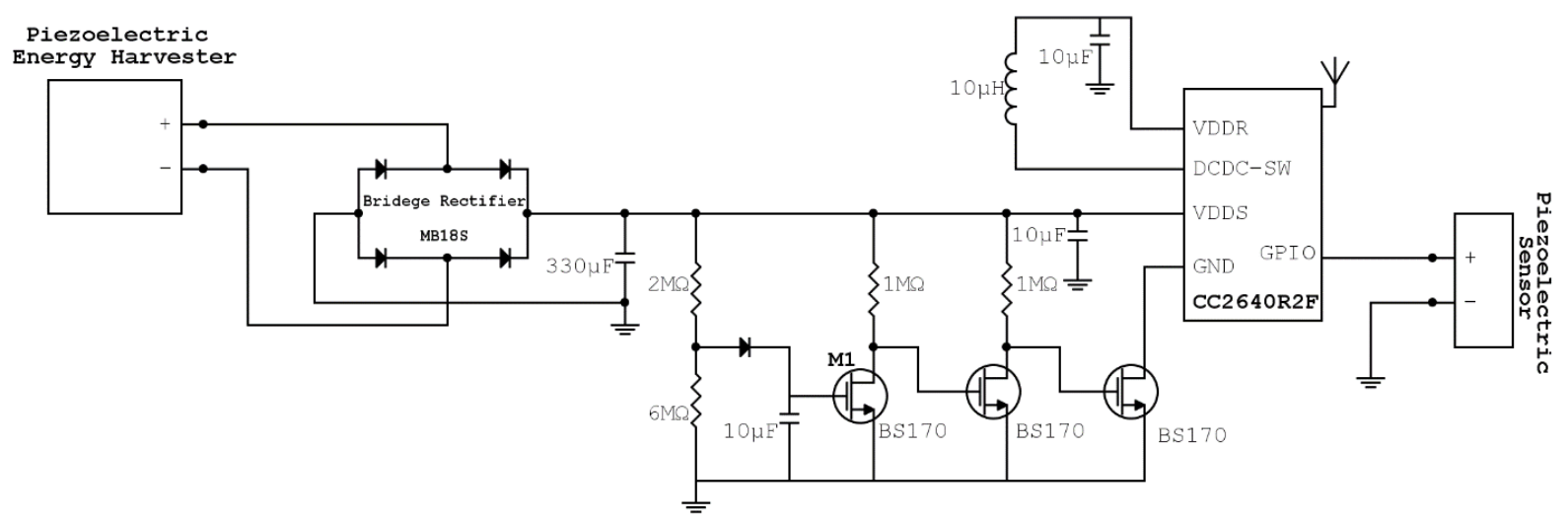

Figure 3.14- Design II circuit schematic

\subsubsection{Embedded software development}

Figure 3.15 shows the process flow chart of the algorithm used to operate the Wireless MCU designed for controlling the wireless sensor node's operation. The process begins by enabling the MCU's active mode through a trigger generated by the voltage detector. At this point, task initialization that includes the followings steps will begin:

- Clearing all the registers

- Pin initialization

- Turning on the GPIO Module (General Purpose Input/Output)

- Selection of the ADC input

- Scheduling the first task execution

- Setting up the ADC parameters

- Setting up the Radio parameters

- Requesting access to ADC

- Requesting access to the Radio 


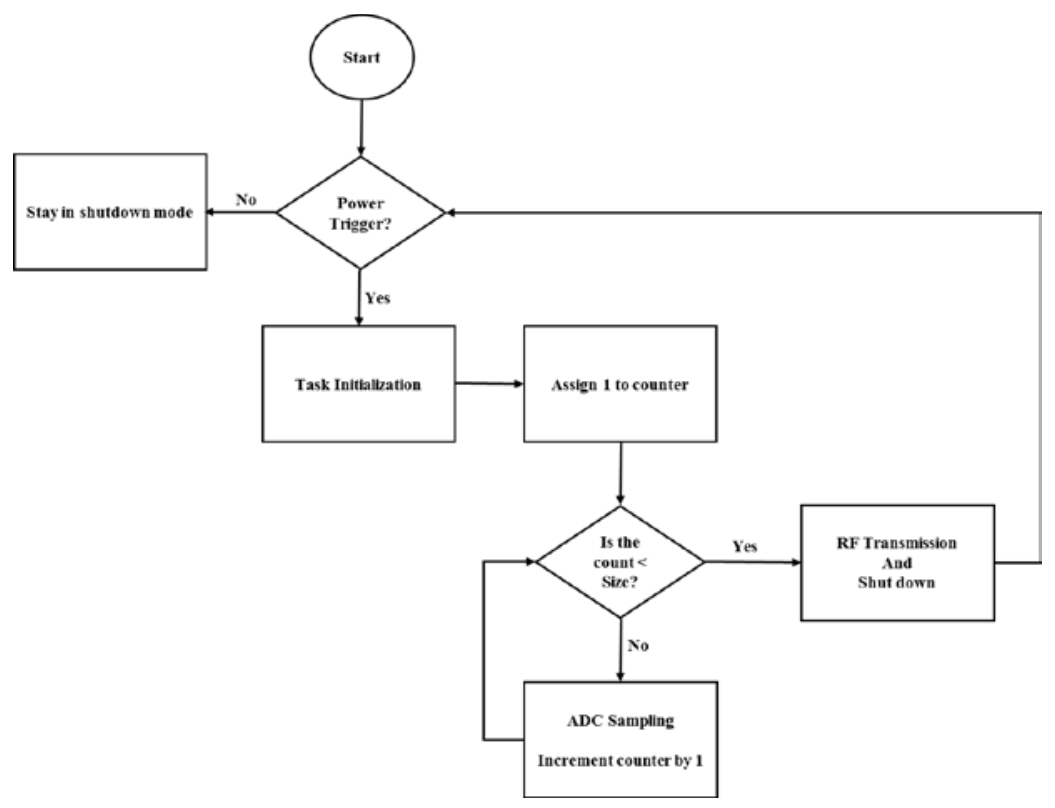

Figure 3.15- Wireless MCU process flow chart

Once task initialization is completed, the ADC module can begin sampling the input signal according to the parameters that were set previously. The ADC is adjusted to obtain 15 samples from the sensor output that are evenly spaced out to cover one complete period of a $60 \mathrm{~Hz}$ signal from the sensor device. Since the ADC's absolute minimum operation voltage is $-0.3 \mathrm{~V}$, the program instructs the ADC to only register samples from the positive portion of the sinusoidal signal (a diode is used to protect the MCU from the negative voltages). The ADC sampling can be triggered using an external timer, $\mathrm{I} / \mathrm{O}$ pins, the software, and the CPU's internal clock. In the case above, sampling is triggered using the interrupts created by the CPU's internal clock (every $111.1 \mu \mathrm{s})$. Once data packets are successfully created and transmitted to the receiver end, ADC and RF modules will be disabled and the device will go to a complete Shutdown mode for maximum power conservation.

\subsubsection{Comparison}

Two design architectures capable of meeting the sensor node's application requirements were introduced in this chapter. Both designs integrate system components according to their specifications so that each part can operate with maximum effectiveness in undertaking its assigned tasks. However, while both designs succeed in providing a functioning self-contained Wireless Sensor Node, they undertake different approaches each with specific advantages and disadvantages. In this section, the two designs will be 
compared based on their merits and the most suitable approach will be selected for the implementation and testing.

Design I focuses on regulating and storing the output energy from the EH before it is used for powering the control and radio devices. Once the storage unit reaches the required 3.3V, the operation of MCU will begin. The MCU will follow its given instruction to achieve the application requirement of the sensor node. Although Design I can remain functional for a longer period after the storage unit is full (due to its larger energy storage capacity), its duty cycle lacks flexibility once the sensor node is mounted in place. The duty cycle of the device is set using a programmable timer and cannot be altered once the unit is assembled. Therefore, the timer must be adjusted according to the worst charging conditions which reduces the operation efficiency of the unit.

Design II takes advantage of power management capabilities of the MCU to develop an intelligent and dynamic system that is capable of adjusting the operating duty cycle of the wireless sensor node according to the performance of the EH. Therefore, the need for the CPU's internal clock or any external clock is eliminated. The unique dynamic characteristic of this design allows the system to be more flexible in its operation as changes take place in the operating conditions surrounding the sensor node. In spite of this unique characteristic that significantly enhances the power consumption efficiency of the unit, due to limited size of the storage capacitor in this design, the sensor node's operation is terminated during power outage periods. Nevertheless, regardless of this drawback, and according to the requirements of the system, it was concluded that design II is a more suitable approach due to its lower power loss, faster initial charging period, and increased frequency of signal transmission.

\subsection{Summary}

According to the system requirements for the electric current monitoring application, two design approaches are developed and introduced in this chapter. Both approaches tackle some of the critical constraints associated with the design of WSNs. Design I focuses on regulating the voltage output of the EH to power the Wireless MCU. In this case, the operating duty cycle lacks flexibility due to the use of a programmable timer. Design II uses a more dynamic approach that is capable of adjusting the operating duty cycle of the sensor node according to the performance of the EH. Taking into consideration the key advantages and disadvantages of each design, Design II is chosen as the more suitable approach for the purpose of this application and will be implemented for further testing and analysis. 


\section{Chapter 4}

\section{Implementation and Testing}

\subsection{Introduction}

This chapter focuses on implementation, testing and analysis of the selected wireless sensor node design (Design II). The unit is tested according to the application requirements for the system indicated in section 2.8. According to these requirements, a number of different testing scenarios were developed to ensure the appropriate performance of the unit under all conditions. Additionally, stress testing was performed so as to confirm the sensor node's stability under abnormal conditions including power outages, disruptions or changes in the current passing through the electric wire, or loss of connection with the central hub.

Once the experimental setup is adjusted to replicate the end-use operating condition of the wireless sensor node, ADC measurements are captured and transmitted from the sensor node. The information obtained on the receiver end are compared to the output signal from the sensor to validate the operation's accuracy. Lastly, the charging and discharging behavior of the storage unit is monitored throughout the 
sensor node's operation. Using the data obtained, the operating duty cycle as a function of current passing through the wire is reported.

\subsection{Experimental Setup and Equipment}

To validate the performance of the designed self-powered wireless sensor node, the EH is mounted at a distance of $6 \mathrm{~mm}$ above a wire connecting an adjustable heater to the electric receptacle. The heater can work under four different operating modes leading to four arbitrary AC currents passing through the wire. The AC current drawn by the heater is measured and reported separately using an AC clamp-on meter (Fluke i400s AC Current Clamp). The Piezoelectric sensor is mounted at a distance of $10 \mathrm{~mm}$ above the wire with its outputs connected to the designated pins on the circuit board. Furthermore, the output signal from the sensor is monitored using a Tektronix TDS 2114C oscilloscope. All required connections to the oscilloscope are made using 10:1 probes with an input impedance of $10 \mathrm{M} \Omega$ and input capacitance of $15 \mathrm{pF}$. Due to the limited performance of the sensor under the accessible testing conditions, an Agilent 33522A waveform generator is used to generate different signals replicating various outputs obtainable from the sensor device.

Code Composer Studio (v7.0.0) software application is used to develop, edit, build, and debug the source code. Once the source file is built, it is loaded onto the Wireless MCU using the SimpleLink ${ }^{\mathrm{TM}}$ CC2640R2 LaunchPad development kit supporting the CC2640R2F Wireless MCU. The loaded MCU is connected to other circuit components and modules following the design schematic provided in Figure 3.14.

The transmitted data packets are received using a CC2650 Wireless MCU LaunchPad ${ }^{\mathrm{TM}}$ Kit. The unit is connected to the Desktop computer using its on-board emulator and USB serial port at a distance of one meter away from the sensor node. SmartRFTM Studio (v2.6.0), a Windows application used to evaluate Low Power RF devices, is used to operate the CC2650 Wireless MCU LaunchPad in order to receive the transmitted data packets and to test and validate the data transmission process.

\subsection{Results and Discussion}

In this section, test results obtained from the performance analysis of the designed self-contained wireless sensor node are provided and discussed. Once the unit is mounted in place, sensor data is transmitted to the receiver hub in order to validate the sensor node's operation. Furthermore, key 
performance parameters such as the initial charging time, operating frequency and duty cycle are presented and their dependency to the EH's performance is investigated.

\subsubsection{Performance Validation}

In order to validate the performance of the wireless sensor node, the transmitted ADC values from the sensor node were compared to the sensor's output captured by the oscilloscope. According to the specified application requirements, the unit must be able to capture the peak voltage value of the sensor's $60 \mathrm{~Hz}$ sinusoidal signal within $10 \%$ of its error range. In Figure 4.1, the captured data points at the receiver node are plotted against the reference signal obtained using the oscilloscope. The graph only shows the positive portion of a single cycle (seven evenly spaced data points that were read from a positive half cycle of a signal). Furthermore, the phases are aligned for better representation of the results. The sensor's signal in this case has a peak value of $0.8 \mathrm{~V}$ as measured by the oscilloscope.

The captured ADC point with the highest value among the seven transmitted data points from the sensor node has a voltage value of $0.750 \mathrm{~V}$. This peak value has a nominal error of $-6.25 \%$. With this error, the transmitted peak value by the sensor node lies within the $10 \%$ error range of the actual peak value and therefore, validates the sensor node’s operation.
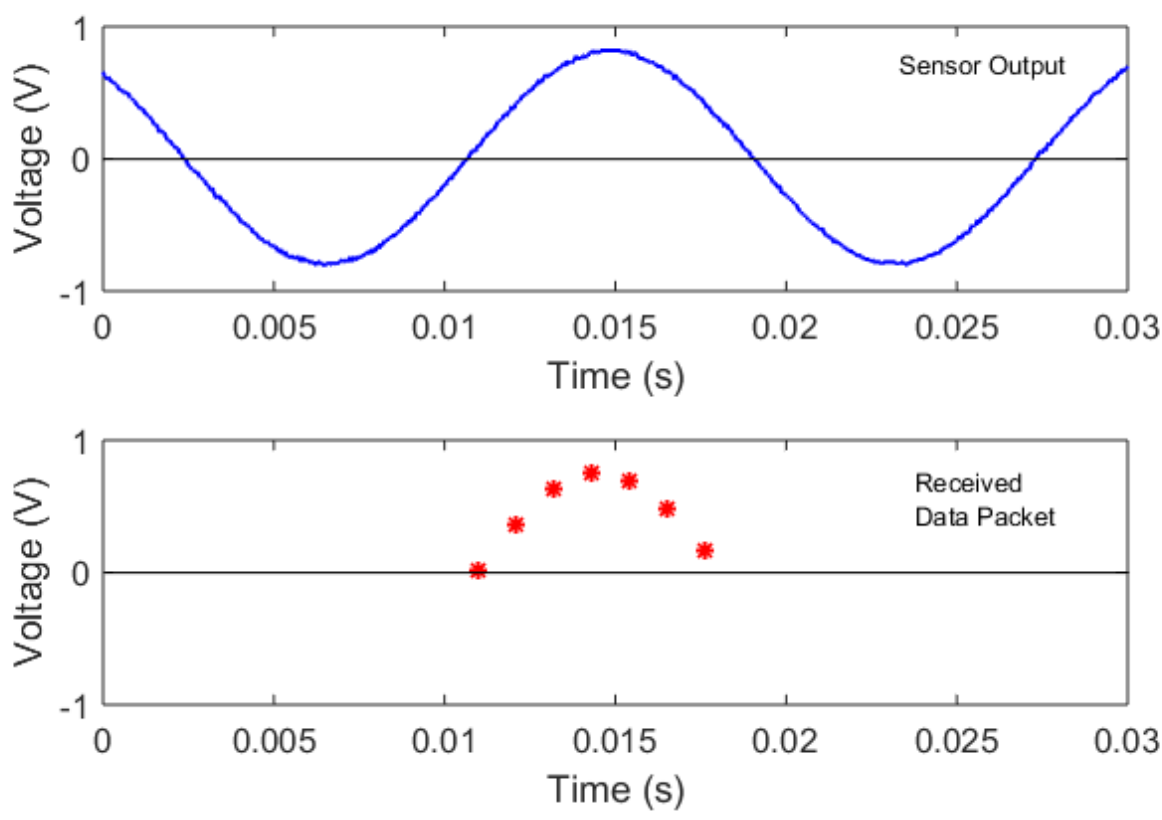

Figure 4.1 - Sensor output signal and the transmitted ADC values 
In order to verify the consistency and reliability of the device's operation over time, multiple signals replicating the sensor's output (generated using a function generator) are fed into the sensor node and the transmitted ADC values are captured and compared to the peak voltage values of the input signal. In each case, the peak voltage obtained from the measurement is compared to the real peak value of the signal and error percentage associated with the measurement is calculated to further validate the performance of the unit. Two of these measurements are provided in Table 4.1 as samples in order to familiarize the reader with the peak detection process.

As indicated in Table 4.1, the error percentage associated with both measurement are within the acceptable range for this project. It is important to note that this error is caused by the limited number of points available from discrete sampling to cover the sensor's signal. With only 15 points representing a full cycle of the sensor's signal, a worst case error of $-8.56 \%$ is expected as the result.

Table 4.1-Transmitted ADC values for two arbitrary signal inputs to the wireless sensor node

\begin{tabular}{|c|}
\hline $\begin{array}{c}\text { Captured ADC values for a } \\
\text { 60Hz, 0.36V pk-pk signal }\end{array}$ \\
\hline 0.04 \\
\hline 0.096 \\
\hline 0.144 \\
\hline 0.172 \\
\hline 0.175 \\
\hline 0.152 \\
\hline 0.11 \\
\hline 0.054 \\
\hline 0.001 \\
\hline 0.047 \\
\hline 0.103 \\
\hline 0.149 \\
\hline 0.175 \\
\hline 0.171 \\
\hline 0.143 \\
\hline Calculated Signal Peak: $0.175 \mathrm{~V}$ \\
\hline Nominal Error: $-2.7 \%$ \\
\hline
\end{tabular}

\begin{tabular}{|c|}
\hline $\begin{array}{c}\text { Captured ADC values for a } \\
\text { 60Hz, 1.0V pk-pk signal }\end{array}$ \\
\hline 0.202 \\
\hline 0.377 \\
\hline 0.465 \\
\hline 0.446 \\
\hline 0.327 \\
\hline 0.130 \\
\hline 0.008 \\
\hline 0.225 \\
\hline 0.391 \\
\hline 0.468 \\
\hline 0.438 \\
\hline 0.301 \\
\hline 0.099 \\
\hline 0.015 \\
\hline 0.232 \\
\hline Calculated Signal Peak: $0.468 \mathrm{~V}$ \\
Nominal Error: -6.4\% \\
\hline \\
\hline
\end{tabular}




\subsubsection{Transmission Duty Cycle Analysis}

An important characteristic of self-contained wireless sensor nodes is their duty cycle and operating frequency. This duty cycle is defined as the fraction of one period in which the sensor node is in active mode. A lower duty cycle corresponds to a longer time lapse between two successive read/transmit operations. Therefore, as the duty cycle increases, data packets are transmitted more frequently to the central hub providing the user with more information from the sensor node. In most cases, the operating duty cycle of all nodes is fixed across the Wireless Sensor Network and it is set by the MCU's clock according to the requirements of the end user.

In the case of design presented in this work, the frequency of operation of the wireless sensor node is adjusted according to the performance of the EH. Such unique characteristic allows the sensor node to be more flexible in its operation as changes take place in the operating conditions. In order to enhance the operation effectiveness, the focus is placed on reducing the time lapse between two successive read/transmit operations. This period corresponds to the time in which the sensor node accumulates enough energy to perform a single ADC measurement and data transmission. As this latency period is reduced (increased frequency of operation), more data packet containing information from the sensor node are transmitted to the user over time.

Another important characteristic of a self-contained sensor node is its initial charging period which is defined as the time it takes for a node with an unfilled storage unit to accumulates enough energy to complete its very first read and transmit operation. As shown in Figure 4.2, as the wireless sensor node is placed on a wire conducting a 7.6A current, it takes 242 seconds for the storage unit to reach $3 \mathrm{~V}$ and to activate the trigger initiating the Wireless MCU's operation. Upon completion of the first transmission, the voltage across the storage unit drops and the MCU returns to the Shutdown mode until it re-accumulates enough energy to send the consecutive data packet (recharging period).

The total MCU active time for ADC measurement and wireless transmission is measured to be approximately $4.8 \mathrm{~ms}$. Using this value, the frequency of operation as a function of current passing through the wire can be calculated. In the case of the sensor node placed on a wire conducting a current of 7.6A, the operation duty cycle is calculated to be $0.00315 \%$. It is important to note that this is the operation duty cycle under the worst energy harvesting condoning (the current passing through the wire is at its minimum value within the given application range). The frequency of operation is expected to increase as the intensity of the current passing through the wire is increased (the storage unit can reach the $3 \mathrm{~V}$ voltage faster due to the higher EH's power output as the intensity of current passing through the wire is increased). 


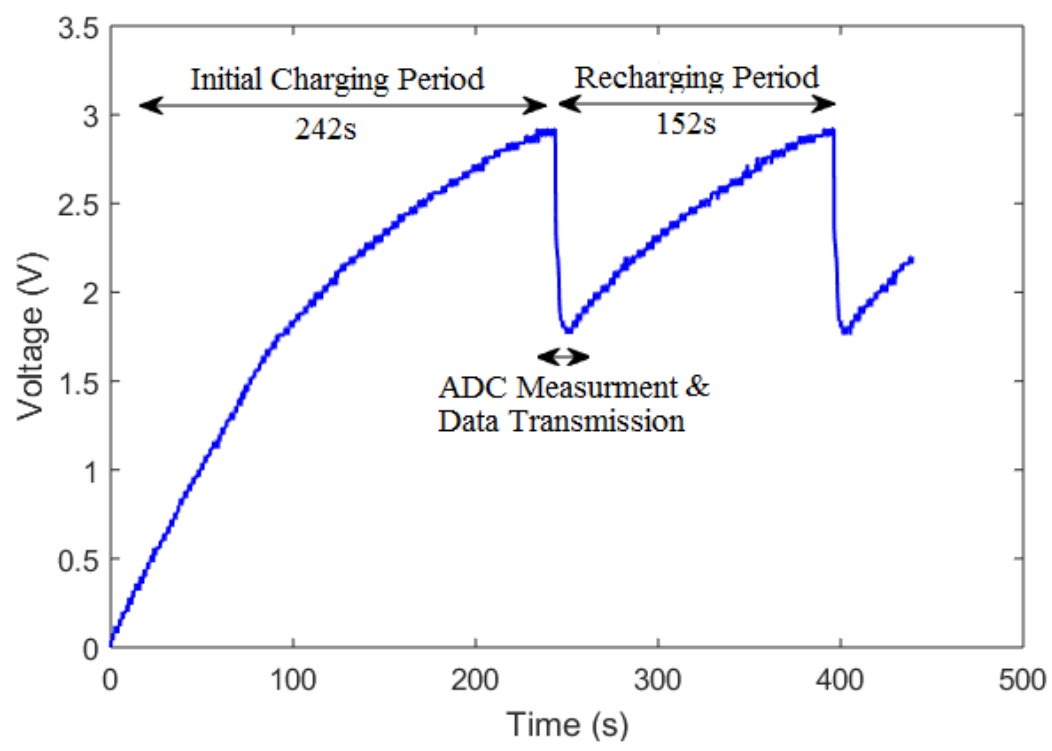

Figure 4.2- Charging/Discharging behavior of the Storage Unit as data packets are transmitted from the sensor node when the wire is conducting a current of 7.6A.

In order to better understand the effect the EH's power output on the sensor node's operation, the charging and discharging behavior of the storage unit is monitored as the wireless sensor node is place on a wire conducting three arbitrary currents within the application requirement range (11.8A and 19.4A and also 25.7 A). Results of this experiment are shown in Figure 4.3 and are summarized in Table 4.2.

As expected, the initial charging period and also the recharging time lapse are reduced as the intensity of the current passing through the wire is increased. However, it is observed that for currents above 20A, the frequency of signal transmission does not increase substantially. Moreover, the voltage across the storage unit exceeds $3 \mathrm{~V}$ before the trigger circuit activates the MCU and its ADC module. This phenomenon can be explained by the effect of the Voltage Detector's RC time constant.

Table 4.2- Duty Cycle of operation as a function of current passing through the wire

\begin{tabular}{|c|c|c|c|}
\hline $\begin{array}{l}\text { Current passing } \\
\text { through the wire (A) }\end{array}$ & $\begin{array}{l}\text { Initial charging } \\
\text { period (s) }\end{array}$ & $\begin{array}{l}\text { Recharging } \\
\text { period (s) }\end{array}$ & Duty Cycle \\
\hline 7.6 & 242.0 & 152.0 & $0.00315 \%$ \\
\hline 11.8 & 167.2 & 99.2 & $0.00483 \%$ \\
\hline 19.4 & 115.0 & 68.4 & $0.00701 \%$ \\
\hline 25.7 & 98.4 & 59.2 & $0.00810 \%$ \\
\hline
\end{tabular}




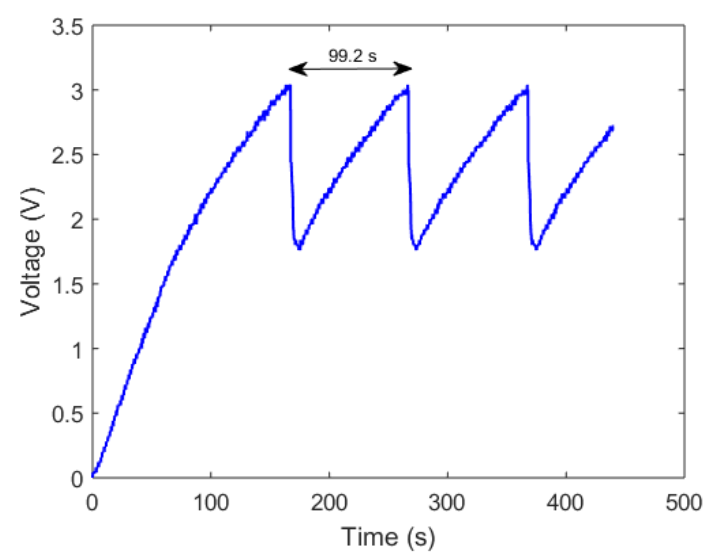

(a)

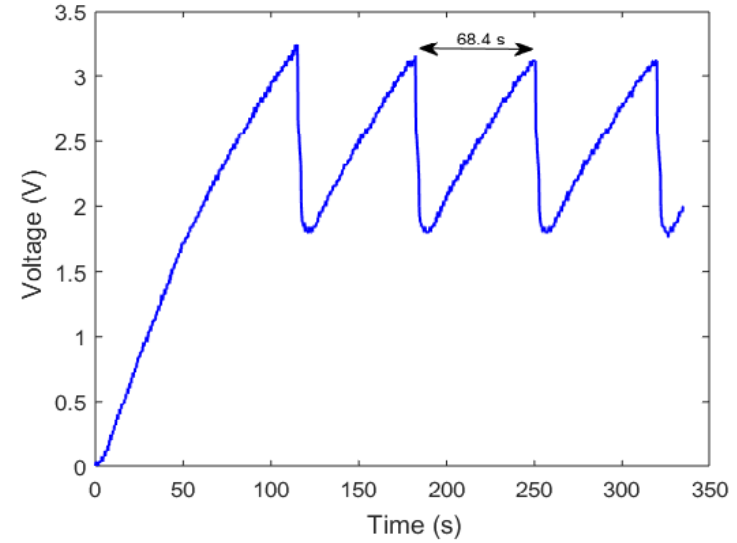

(b)

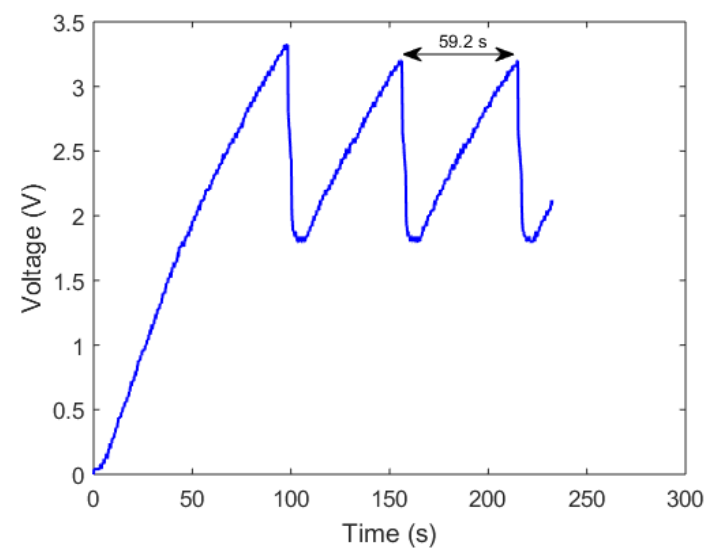

(c)

Figure 4.3- Charging/Discharging behavior of the Storage Unit as data packets are transmitted from the sensor node when the wire is conducting a current of (a) $11.8 \mathrm{~A}$, (b) $19.4 \mathrm{~A}$, (c) $25.7 \mathrm{~A}$

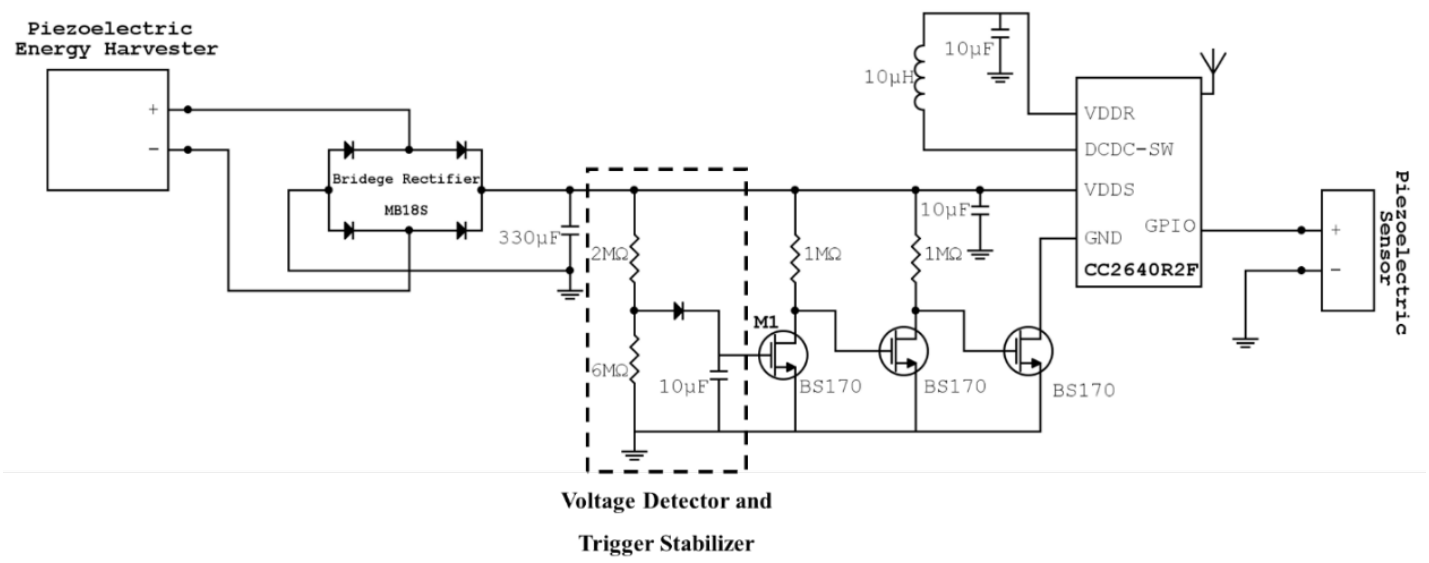

Figure 4.4- Voltage Detector and the Trigger Stabilizer 
As shown in Figure 4.4, the voltage divider in conjugation with the $10 \mu \mathrm{F}$ capacitor are responsible for monitoring the voltage across the super capacitor and triggering the MCU using the multistage amplifier. As the power output of the $\mathrm{EH}$ increases, the $330 \mathrm{uF}$ storage capacitor will reach the $3 \mathrm{~V}$ voltage before the $10 \mathrm{uFcapacitor}$ can reach the $1.75 \mathrm{~V}$ required to trigger the multistage amplifier. Therefore, the operating frequency of the system and the initial charging time of the storage unit will be bounded by the charging time of the Voltage Detector and the Trigger Stabilizer capacitor.

\subsection{Summary}

According to the requirements for the electric current monitoring application, experimental setup is adjusted to replicate the end-use operating condition of the wireless sensor node. Subsequently, a number of different testing scenarios were developed to ensure the appropriate performance of the unit under all conditions. Test results obtained from the performance analysis of the designed self-contained wireless sensor node indicate key performance parameters such as the initial charging time, operating frequency and the duty cycle. As it had been expected, the initial charging period and also the recharging time lapses are reduced as the intensity of the current passing through the wire is increased. Therefore, the frequency of operation increases and data packets are transmitted more frequently to the central hub providing the user with more information from the sensor node. However, it is observed that for currents above 20A, the operation frequency of the sensor node does not increase substantially as the current passing through the wire increases, limited by the Voltage Detector's RC time constant. Lastly, it is demonstrated that the transmitted ADC values from the sensor node are able to able to capture the peak voltage value of the sensor's $60 \mathrm{~Hz}$ sinusoidal signal within $10 \%$ of its error range. The consistency and reliability of this operation is validated by testing the unit with multiple signals generated using a waveform generator and replicating the sensor's output. 


\section{Chapter 5}

\section{Conclusions \& Future Work}

\subsection{Conclusions}

This thesis has reported the design, development, and integration of a self-contained system intended for real-time monitoring of electricity usage within residential and commercial buildings. Consisting of an Energy Harvester (EH), an electric current sensor, and a Wireless Micro-controller Unit (MCU), each building block was examined thoroughly and their behaviors and fundamental characteristics under various operational conditions were reported. It was shown that the integrated unit must operate when mounted on conducting wires carrying a current in the range of 7A to 27A. Furthermore, the unit should capture the peak voltage value of a $60 \mathrm{~Hz}$ sinusoidal signal with an amplitude in the range of $100 \mathrm{mV}$ to $900 \mathrm{mV}$ within its $10 \%$ error range.

Using these requirements as a basis, two designs methodologies were introduced to implement the system modules within the integrated unit according to their specifications while meeting the design 
requirements. A detailed account of each design's blueprint, a complete circuit design as well as the embedded software developed to run the system were provided. While both designs were capable of meeting all the system requirements, each entailed its own advantages and disadvantages. The performance of each design was investigated according to the application requirements as well as performance parameters such as stability as changes take place in the operating conditions surrounding the sensor node, power consumption efficiency, and the sensor node's flexibility in adjusting its operating duty cycle according to the energy generation capabilities of the EH.

Upon preliminary analysis of units in each case, key advantages and disadvantages of each design were identified. Using this information, the design most suitable in meeting the requirements of the system was selected and implemented for testing. The selected design entails unique characteristics such as a dynamic functionality to adapt to changes taking place in the operating environment, increased power consumption efficiency, increased frequency of ADC measurement and data transmission, and flexibility in adjusting the operating duty cycle as the energy generation capabilities of EH varies.

To validate the performance of the designed self-powered wireless sensor node, the testing circuit was implemented and the unit was mounted in place to analyze the performance of the designed self-contained wireless sensor node. It was shown that the unit is capable of capturing the peak voltage value of the sensor's $60 \mathrm{~Hz}$ sinusoidal signal within $8.56 \%$ of its error range (less than the $10 \%$ value indicated in the system requirements).

Furthermore, it was demonstrated that the initial charging period and also the recharging period are reduced as the intensity of the current passing through the wire is increased. The total MCU active time during a single cycle operation was reported to be $4.8 \mathrm{~ms}$. Using this value, the operation duty cycle as a function of current passing through the wire was calculated and reported. The obtained values indicate that the operation duty cycle increases as the intensity of the current passing through the wire is amplified.

According to the test results, it was verified that the initial charging period is reduced from $4 \mathrm{~min}$ to $1.6 \mathrm{~min}$ as the current passing through the conducting wire increases from 7.6A to 25.7A. The system can sustain a duty cycle in the range of $0.00315 \%$ to $0.00810 \%$ depending on the intensity of current passing through the line. Lastly, it was observed and concluded that the operating frequency of the system and the initial charging period of the unit are bounded by the charging time of the voltage detector and trigger stabilizer circuit. 


\subsection{Future Work}

The results of this work point to a number of interesting directions to extend the scope of this project. Future work includes a more rigorous testing of the developed self-contained sensor node in order to verify its operation beyond the operating range requirement of the system. Exploring the stability of the unit over time and the impact of fatigue on its operation is also a possibility for future work. Energy Harvesters can be damaged as a result of long-term use. Damages can lead to a reduced power output from the unit and therefore, it is important to investigate their impact on sensor node’s operation.

The work done in this project also paves the way for taking an additional step in integrating energy harvesting units with reduced footprints with the developed sensor node. In order for the sensor node to be functional when supplied with a less powerful source of energy, additional work must be done to further enhance the power consumption efficiency of the circuit. Finally, the EH, electric current sensor, power conditioning circuit, and the Wireless MCU must all be integrated within a single package. This is the last stage of the development before the unit can be deployed for its end use operation. 


\section{References}

[1] Danilo Porcarelli, Domenico Balsamo, Davide Brunelli, "Perpetual and low-cost power meter for monitoring residential and industrial appliances," in IEEE, Grenoble, 2013.

[2] Eli S. Leland, Richard M. White, Paul K. Wright, "Energy scavenging power sources for household electrical monitoring," in PowerMEMS, 2006.

[3] Igor Paprotny, Eli S. Leland, Richard M. White, Paul K. Wright, "Optimization of a die-sized (10x10x4 mm3) MEMS ac energy scavenger for residential and commercial electricity end-use monitoring," in PowerMEMS, Washington, 2009.

[4] Steven B. Lao, Shamsheer S. Chauhan , Tim E. Pollock, Thorben Schröder , In Sik Cho, Armaghan Salehian , "Design, Fabrication and Temperature Sensitivity Testing of a Miniature Piezoelectric-Based Sensor for Current Measurements," Actuators, vol. 3, pp. 162-181, 2014.

[5] M.A. Matin, M.M. Islam , "Overview of Wireless Sensor Network," in Wireless Sensor Networks Technology and Protocols, INTECH, 2012.

[6] Egon Fernandes, Sid Zarabi, Hélène Debéda, Claude Lucat, David Nairn, Lan Wei, Armaghan Salehian, "Modelling and fabrication of a compliant centrally supported meandering piezoelectric energy harvester using screen-printing technology," in IOP Publishing, Journal of Physics, Paris, 2016.

[7] Igor Paprotny, Qiliang Xu, Wai Wah Chan, Richard M. White, Paul K. Wright, "Electromechanical Energy Scavenging from Current-Carrying Conductors," IEEE Sensors Journal, vol. 13, no. 1, 2013.

[8] Shashank Priya, Daniel J. Inman, Energy Harvesting Technologies, New York: Springer Science, 2009.

[9] T. E. Pollock, "Testing of a Miniature Piezoelectric-based EMF Energy Harvester," Waterloo, 2014.

[10] T. H. Ng W. H. Liao, "Sensitivity Analysis and Energy Harvesting for a Self-Powered Piezoelectric Sensor," Journal of Intelligent Material Systems and Structures, vol. 16, 2005. 
[11] E Fernandes, S Zarabi, H Debéda, C Lucat, D Nairn, L Wei and A Salehian, "Modelling and fabrication of a compliant centrally supported meandering piezoelectric energy harvester using screen printing technology," in Journal of Physics: Conference Series, Paris, 2006.

[12] Geffrey K. Ottman ,Heath F. Hofmann, "Adaptive Piezoelectric Energy Harvesting Circuit for Wireless Remote Power Supply," IEEE Transactions on Power Electronics, vol. 17, no. 5, 2002.

[13] Anantha P. Chandrakasan, Denis C. Daly, Joyce Kwong, Yogesh K. Ramadass, "Next Generation Micro-power Systems," in 2008 IEEE Symposium on VLSI Circuits, Honolulu, 2008.

[14] Gyorgy D. Szarka ; Bernard H. Stark ; Stephen G. Burrow, "Review of Power Conditioning for Kinetic Energy Harvesting Systems," IEEE Transactions on Power Electronics, vol. 27, no. 2, pp. $803-815,2011$.

[15] Frederick L. Burghardt, Andrew C. Waterbury, Igor Paprotny, Lindsay M. Miller, Peter Minor, Rafael Send, Qiliang Xu, Richard M. White, and Paul K. Wright, "A Design Methodology for Energy Harvesting With a Case Study on the Structured Development of a System to Power a Condition Monitoring Unit," Energy Harvesting and Systems, vol. 1, pp. 101-112, 2014.

[16] D. P. Arnold, "Review of Microscale Magnetic Power Generation," IEEE Transactions on Magnetics, vol. 43, no. 11, 2007.

[17] Yat-Hei Lam, Wing-Hung Ki, Chi-Ying Tsui,, "Integrated Low-Loss CMOS Active Rectifier for Wirelessly Powered Devices," IEEE Transactions on Circuits and Systems, vol. 53, no. 12, 2006.

[18] C. Peters, O. Kessling, F. Henrici, M. Ortmanns, Y. Manoli, "CMOS Integrated Highly Efficient Full Wave Rectifier," in IEEE: Circuits and Systems, New Orleans, 2007.

[19] R Torah, P Glynne-Jones, M Tudor, T O’Donnell, S Roy, S Beeby, "Self-powered autonomous wireless sensor node using vibration energy harvesting," Measurement Science and Technology, vol. 19, no. 12, 2008.

[20] J. Colomer ; J. Brufau ; P. Miribel-Catala ; A. Saiz-Vela ; M. Puig-Vidal ; J. Samitier, "Power conditioning circuitry for a self-powered mobile system based on an array of micro PZT generators in a $0.13 \mu \mathrm{m}$ technology," in IEEE: Industrial Electronics, Vigo, 2007. 
[21] Chi-ying Tsui ; Hui Shao ; Wing-Hung Ki ; Feng Su, "Ultra-low voltage power management and computation methodology for energy harvesting applications," in IEEE: VLSI Circuits, Kyoto, 2005.

[22] Dibin Zhu, Stephen P. Beeby, Michael J. Tudor, Nick R. Harris, "A credit card sized self powered smart sensor node," Sensors and Actuators A: Physical, vol. 169, pp. 317-325, 2011.

[23] N. Stephen, "On energy harvesting from ambient vibration," Journal of Sound and Vibration, vol. 293, no. 1, pp. 409-425, 2006.

[24] W J Wu, A M Wickenheiser, T Reissman, E Garcia, "Modeling and experimental verification of synchronized discharging techniques for boosting power harvesting from piezoelectric transducers," Smart Materials and Structures, vol. 18, no. 5, 2009.

[25] Silvio Ziegler, Robert C. Woodward, Herbert Ho-Ching Iu, Lawrence J. Borle, "Current Sensing Techniques: A Review," IEEE SENSORS JOURNAL, vol. 9, no. 4, 2009.

[26] I. Paprotny, E. Leland, C. Sherman, R. M. White and P. K. Wright, "Self-powered MEMS sensor module for measuring electrical quantities in residential, commercial, distribution and transmission power systems," in Energy Conversion Congress and Exposition (ECCE), Atlanta, 2010.

[27] Q. Xu, M. Seidel, I. Paprotny, R. M. White and P. K. Wright, "Integrated centralized electric current monitoring system using wirelessly enabled non-intrusive AC current sensors," in IEEE: Sensors, Limerick, 2011.

[28] Konstantin Mikhaylov ; Jouni Tervonen, "Optimization of microcontroller hardware parameters for Wireless Sensor Network node power consumption and lifetime improvement," in IEEE: Ultra Modern Telecommunications and Control Systems and Workshops (ICUMT), Moscow, 2010.

[29] Diego Antolín, Nicolás Medrano, Belén Calvo, Francisco Pérez, "A Wearable Wireless Sensor Network for Indoor Smart Environment Monitoring in Safety Applications," Sensors, vol. 17, no. 2, 2017.

[30] Jacob Borgeson, Stefan Schauer, Horst Diewald, "Benchmarking MCU power consumption for ultra-low-power applications," Texas Instruments, 2012. 
[31] Dejan Raskovic, David Giessel, "Dynamic Voltage and Frequency Scaling For On-Demand Performance and Availability of Biomedical Embedded Systems," IEEE Transactions on Information Technology in Biomedicine, vol. 13, no. 6, 2009.

[32] I. D. Albu, "On-node processing: Algorithms for Activity Classification and Monitoring in Wireless Sensor Networks," Eindhoven University of Technology, Eindhoven, 2009.

[33] Digvijay Singh; Sai Prashanth S.; Sujan Kundu; Ajit Pal, "Low-power Microcontroller for Wireless Sensor Networks," in TENCON 2009 - 2009 IEEE Region 10 Conference, Singapore, 2009.

[34] C. Guy, "Wireless sensor networks," in SPIE 6357, Sixth International Symposium on Instrumentation and Control Technology, Beijing, 2006.

[35] F. L. Lewis, "Wireless Sensor Networks," in Smart Environments: Technologies, Protocols, and Applications, New York, 2004.

[36] "conceptdraw," CS Odessa Corp., 2017. [Online]. Available:

http://www.conceptdraw.com/examples/topologies. [Accessed 255 2017].

[37] G. Reiter, "Wireless connectivity for the Internet of Things," Texas Instruments Incorporated, Texas, 2014.

[38] W. H. Hayt, Engineering Circuit Analysis, Mcgraw-Hill College Division, 1993.

[39] "CC2640R2F SimpleLink ${ }^{\mathrm{TM}}$ Bluetooth ${ }^{\circledR}$ low energy Wireless MCU," Texas Instrument Incorporated, Dallas, 2016.

[40] "ATmega640/V-1280/V-1281/V-2560/V-2561/V Datasheet," Atmel Corporation, San Jose, 2014.

[41] V. Raghunathan ; C. Schurgers ; Sung Park ; M.B. Srivastava, "Energy-aware wireless microsensor networks," IEEE Signal Processing Magazine, vol. 19, no. 2, pp. 40 - 50, 2002. 